\title{
AN ANALYTICAL MODEL FOR PROGNOSIS OF MASS TRANSPORT DURING MANUFACTURING OF A BIT-CELL WITHOUT READ-PORT TRANSISTORS TO INCREASE INTEGRATION RATE OF ELEMENTS OF THE CIRCUIT
}

\author{
E. L. Pankratov \\ Nizhny Novgorod State University, 23 Gagarin Avenue, Nizhny Novgorod, \\ 603950, Russia \\ Nizhny Novgorod State Technical University, 24 Minin Street, Nizhny \\ Novgorod, 603950, Russia
}

\begin{abstract}
In this paper, we introduce an analytical approach for prognosis of mass transport during manufacturing of a bit-cell circuit without read-port transistors. Based on this approach we obtain conditions to increase density of elements of this circuit, manufactured by diffusion or ion implantation with optimized annealing time of dopant and/or radiation defects. The above analytical approach gives a possibility to take into account nonlinearity of the mass transport, dependences of parameters of the transport on spatial coordinate and time.
\end{abstract}

${ }^{*}$ Corresponding author.

E-mail address: elp2004@mail.ru (E. L. Pankratov).

Copyright @ 2020 Scientific Advances Publishers 2020 Mathematics Subject Classification: 35A20.

Submitted by Li Li.

Received November 8, 2019 
Keywords: partial differential equation, analytical approach for solution, taking into account nonlinearity of the mass transport, dependences of parameters of the transport on spatial coordinate and time, prognosis of mass transport, manufacturing of a bit-cell circuit, increasing of density of elements of this circuit.

\section{Introduction}

An actual and intensively solving problems of solid state electronics is increasing of integration rate of elements of integrated circuits ( $p$ - $n$-junctions, their systems et al) [1-8]. Increasing of the integration rate leads to necessity to decrease their dimensions. To decrease the dimensions are using several approaches. They are widely using laser and microwave types of annealing of infused dopants. These types of annealing are also widely using for annealing of radiation defects, generated during ion implantation [9-17]. Using the approaches gives a possibility to increase integration rate of elements of integrated circuits through inhomogeneity of technological parameters due to generating inhomogenous distribution of temperature. In this situation one can obtain decreasing dimensions of elements of integrated circuits [18] with account Arrhenius law [1, 3]. Another approach to manufacture elements of integrated circuits with smaller dimensions is doping of heterostructure by diffusion or ion implantation [1-3]. However in this case optimization of dopant and/or radiation defects is required [18].

In this paper we consider a heterostructure. The heterostructure consist of a substrate and several epitaxial layers. Some sections have been manufactured in the epitaxial layers. Further we consider doping of these sections by diffusion or ion implantation. The doping gives a possibility to manufacture field-effect transistors framework an integrator circuit so as it is shown on Figure 1. The manufacturing gives a possibility to increase density of elements of circuit of the bit-cell without read-port transistors [4]. After the considered doping dopant 
and/or radiation defects should be annealed. Framework the paper we analyzed dynamics of redistribution of dopant and/or radiation defects during their annealing. We introduce an approach to decrease dimensions of the element. However it is necessary to complicate technological process.

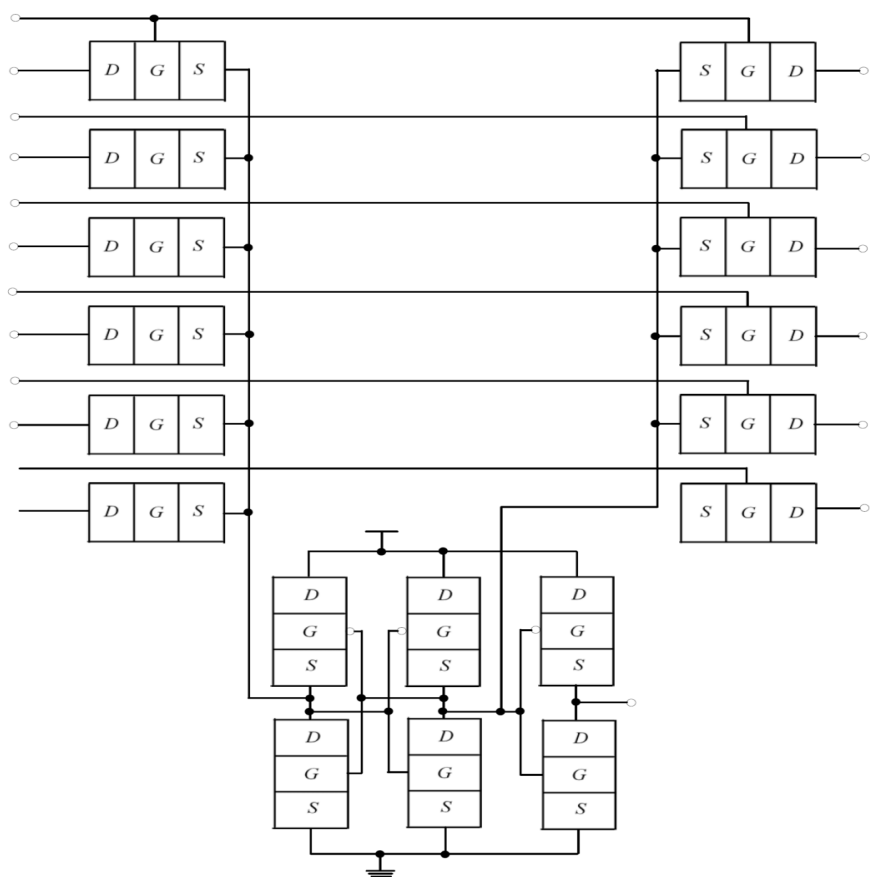

Figure 1. The considered bit-cell circuit [4]. 


\section{Method of Solution}

In this section we determine spatio-temporal distributions of concentrations of infused and implanted dopants. To determine these distributions, we calculate appropriate solutions of the second Fick's law $[1,3,18]$

$$
\begin{aligned}
\frac{\partial C(x, y, z, t)}{\partial t}=\frac{\partial}{\partial x}\left[D_{C} \frac{\partial C(x, y, z, t)}{\partial x}\right] & +\frac{\partial}{\partial y}\left[D_{C} \frac{\partial C(x, y, z, t)}{\partial y}\right] \\
& +\frac{\partial}{\partial z}\left[D_{C} \frac{\partial C(x, y, z, t)}{\partial z}\right] .
\end{aligned}
$$

Boundary and initial conditions for the equations are

$$
\begin{gathered}
\left.\frac{\partial C(x, y, z, t)}{\partial x}\right|_{x=0}=0,\left.\quad \frac{\partial C(x, y, z, t)}{\partial x}\right|_{x=L_{x}}=0,\left.\quad \frac{\partial C(x, y, z, t)}{\partial y}\right|_{y=0}=0 \\
\left.\frac{\partial C(x, y, z, t)}{\partial y}\right|_{y=L_{y}}=0,\left.\quad \frac{\partial C(x, y, z, t)}{\partial z}\right|_{z=0}=0,\left.\quad \frac{\partial C(x, y, z, t)}{\partial z}\right|_{z=L_{z}}=0 \\
C(x, y, z, 0)=f(x, y, z)
\end{gathered}
$$

The function $C(x, y, z, t)$ describes the spatio-temporal distribution of concentration of dopant; $T$ is the temperature of annealing; $D_{C}$ is the dopant diffusion coefficient. Value of dopant diffusion coefficient could be changed with changing materials of heterostructure, with changing temperature of materials (including annealing), with changing concentrations of dopant and radiation defects. We approximate dependences of dopant diffusion coefficient on parameters by the following relation with account results in [20-22]:

$$
\begin{aligned}
D_{C}=D_{L}(x, y, z, T) & {\left[1+\xi \frac{C^{\gamma}(x, y, z, t)}{P^{\gamma}(x, y, z, T)}\right] } \\
& \times\left[1+\varsigma_{1} \frac{V(x, y, z, t)}{V^{*}}+\varsigma_{2} \frac{V^{2}(x, y, z, t)}{\left(V^{*}\right)^{2}}\right] .
\end{aligned}
$$


Here the function $D_{L}(x, y, z, T)$ describes the spatial (in heterostructure) and temperature (due to Arrhenius law) dependences of diffusion coefficient of dopant. The function $P(x, y, z, T)$ describes the limit of solubility of dopant. Parameter $\gamma \in[1,3]$ describes average quantity of charged defects interacted with atom of dopant [20]. The function $V(x, y, z, t)$ describes the spatio-temporal distribution of concentration of radiation vacancies. Parameter $V^{*}$ describes the equilibrium distribution of concentration of vacancies. The considered concentrational dependence of dopant diffusion coefficient has been described in details in [20]. It should be noted, that using diffusion type of doping did not generation radiation defects. In this situation $\zeta_{1}=\zeta_{2}=0$. We determine spatio-temporal distributions of concentrations of radiation defects by solving the following system of equations [21, 22]:

$$
\begin{aligned}
\frac{\partial I(x, y, z, t)}{\partial t}= & \frac{\partial}{\partial x}\left[D_{I}(x, y, z, T) \frac{\partial I(x, y, z, t)}{\partial x}\right] \\
& +\frac{\partial}{\partial y}\left[D_{I}(x, y, z, T) \frac{\partial I(x, y, z, t)}{\partial y}\right] \\
& +\frac{\partial}{\partial z}\left[D_{I}(x, y, z, T) \frac{\partial I(x, y, z, t)}{\partial z}\right] \\
& -k_{I, V}(x, y, z, T) I(x, y, z, t) V(x, y, z, t) \\
& -k_{I, I}(x, y, z, T) I^{2}(x, y, z, t), \\
\frac{\partial V(x, y, z, t)}{\partial t}= & \frac{\partial}{\partial x}\left[D_{V}(x, y, z, T) \frac{\partial V(x, y, z, t)}{\partial x}\right] \\
& +\frac{\partial}{\partial y}\left[D_{V}(x, y, z, T) \frac{\partial V(x, y, z, t)}{\partial y}\right] \\
& +\frac{\partial}{\partial z}\left[D_{V}(x, y, z, T) \frac{\partial V(x, y, z, t)}{\partial z}\right] \\
& -k_{I, V}(x, y, z, T) I(x, y, z, t) V(x, y, z, t) \\
& +k_{V, V}(x, y, z, T) V^{2}(x, y, z, t) .
\end{aligned}
$$


Boundary and initial conditions for these equations are

$$
\begin{gathered}
\left.\frac{\partial \rho(x, y, z, t)}{\partial x}\right|_{x=0}=0,\left.\quad \frac{\partial \rho(x, y, z, t)}{\partial x}\right|_{x=L_{x}}=0,\left.\quad \frac{\partial \rho(x, y, z, t)}{\partial y}\right|_{y=0}=0 \\
\left.\frac{\partial \rho(x, y, z, t)}{\partial y}\right|_{y=L_{y}}=0,\left.\quad \frac{\partial \rho(x, y, z, t)}{\partial z}\right|_{z=0}=0,\left.\quad \frac{\partial \rho(x, y, z, t)}{\partial z}\right|_{z=L_{z}}=0 \\
\rho(x, y, z, 0)=f_{\rho}(x, y, z)
\end{gathered}
$$

Here $\rho=I, V$. The function $I(x, y, z, t)$ describes the spatiotemporal distribution of concentration of radiation interstitials; $D_{\rho}(x, y, z, T)$ are the diffusion coefficients of point radiation defects; terms $V^{2}(x, y, z, t)$ and $I^{2}(x, y, z, t)$ correspond to generation divacancies and diinterstitials; $k_{I, V}(x, y, z, T)$ is the parameter of recombination of point radiation defects; and $k_{I, I}(x, y, z, T)$ and $k_{V, V}(x, y, z, T)$ are the parameters of generation of simplest complexes of point radiation defects.

Further we determine distributions in space and time of concentrations of divacancies $\Phi_{V}(x, y, z, t)$ and diinterstitials $\Phi_{I}(x, y, z, t)$ by solving the following system of equations [21, 22]:

$$
\begin{aligned}
\frac{\partial \Phi_{I}(x, y, z, t)}{\partial t}= & \frac{\partial}{\partial x}\left[D_{\Phi I}(x, y, z, T) \frac{\partial \Phi_{I}(x, y, z, t)}{\partial x}\right] \\
& +\frac{\partial}{\partial y}\left[D_{\Phi I}(x, y, z, T) \frac{\partial \Phi_{I}(x, y, z, t)}{\partial y}\right] \\
& +\frac{\partial}{\partial z}\left[D_{\Phi I}(x, y, z, T) \frac{\partial \Phi_{I}(x, y, z, t)}{\partial z}\right] \\
& +k_{I, I}(x, y, z, T) I^{2}(x, y, z, t) \\
& -k_{I}(x, y, z, T) I(x, y, z, t)
\end{aligned}
$$




$$
\begin{aligned}
\frac{\partial \Phi_{V}(x, y, z, t)}{\partial t}= & \frac{\partial}{\partial x}\left[D_{\Phi V}(x, y, z, T) \frac{\partial \Phi_{V}(x, y, z, t)}{\partial x}\right] \\
& +\frac{\partial}{\partial y}\left[D_{\Phi V}(x, y, z, T) \frac{\partial \Phi_{V}(x, y, z, t)}{\partial y}\right] \\
& +\frac{\partial}{\partial z}\left[D_{\Phi V}(x, y, z, T) \frac{\partial \Phi_{V}(x, y, z, t)}{\partial z}\right] \\
& +k_{V, V}(x, y, z, T) V^{2}(x, y, z, t) \\
& -k_{V}(x, y, z, T) V(x, y, z, t) .
\end{aligned}
$$

Boundary and initial conditions for these equations are

$$
\begin{gathered}
\left.\frac{\partial \Phi_{\rho}(x, y, z, t)}{\partial x}\right|_{x=0}=0,\left.\quad \frac{\partial \Phi_{\rho}(x, y, z, t)}{\partial x}\right|_{x=L_{x}}=0,\left.\quad \frac{\partial \Phi_{\rho}(x, y, z, t)}{\partial y}\right|_{y=0}=0 \\
\left.\frac{\partial \Phi_{\rho}(x, y, z, t)}{\partial y}\right|_{y=L_{y}}=0,\left.\quad \frac{\partial \Phi_{\rho}(x, y, z, t)}{\partial z}\right|_{z=0}=0,\left.\quad \frac{\partial \Phi_{\rho}(x, y, z, t)}{\partial z}\right|_{z=L_{z}}=0 \\
\Phi_{I}(x, y, z, 0)=f_{\Phi I}(x, y, z), \Phi_{V}(x, y, z, 0)=f_{\Phi V}(x, y, z)
\end{gathered}
$$

Here $D_{\Phi \rho}(x, y, z, T)$ are the diffusion coefficients of the above complexes of radiation defects; and $k_{I}(x, y, z, T)$ and $k_{V}(x, y, z, T)$ are the parameters of decay of these complexes.

We calculate distributions of concentrations of point radiation defects in space and time by recently elaborated approach [18]. The approach based on transformation of approximations of diffusion coefficients in the following form: $D_{\rho}(x, y, z, T)=D_{0 \rho}\left[1+\varepsilon_{\rho} g_{\rho}(x, y, z, T)\right]$, where $D_{0 \rho}$ are the average values of diffusion coefficients, $0 \leq \varepsilon_{\rho}<1,\left|g_{\rho}(x, y, z, T)\right| \leq 1$, $\rho=I, V$. We also used analogous transformation of approximations of parameters of recombination of point defects and parameters of generation of their complexes: $k_{I, V}(x, y, z, T)=k_{0 I, V}\left[1+\varepsilon_{I, V} g_{I, V}(x, y, z, T)\right]$, 
$k_{I, I}(x, y, z, T)=k_{0 I, I}\left[1+\varepsilon_{I, I} g_{I, I}(x, y, z, T)\right]$ and $k_{V, V}(x, y, z, T)=k_{0 V, V}$ $\left[1+\varepsilon_{V, V} g_{V, V}(x, y, z, T)\right]$, where $k_{0 \rho 1, \rho 2}$ are the their average values, $0 \leq \varepsilon_{I, V}<1,0 \leq \varepsilon_{I, I}<1,0 \leq \varepsilon_{V, V}<1,\left|g_{I, V}(x, y, z, T)\right| \leq 1,\left|g_{I, I}(x, y, z, T)\right| \leq 1$, $\left|g_{V, V}(x, y, z, T)\right| \leq 1$. Let us introduce the following dimensionless variables: $\widetilde{I}(x, y, z, t)=I(x, y, z, t) / I^{*}, \tilde{V}(x, y, z, t)=V(x, y, z, t) / V^{*}$, $\omega=L^{2} k_{0 I, V} / \sqrt{D_{0 I} D_{0 V}}, \Omega_{\rho}=L^{2} k_{0 \rho, \rho} / \sqrt{D_{0 I} D_{0 V}}, \vartheta=\sqrt{D_{0 I} D_{0 V}} t / L^{2}$, $\chi=x / L_{x}, \eta=y / L_{y}, \phi=z / L_{z}$. The introduction leads to transformation of Equations (4) and conditions (5) to the following form:

$$
\begin{aligned}
\frac{\partial \tilde{I}(\chi, \eta, \phi, \vartheta)}{\partial \vartheta}= & \frac{D_{0 I}}{\sqrt{D_{0 I} D_{0 V}}} \frac{\partial}{\partial \chi}\left\{\left[1+\varepsilon_{I} g_{I}(\chi, \eta, \phi, T)\right] \frac{\partial \tilde{I}(\chi, \eta, \phi, \vartheta)}{\partial \chi}\right\} \\
& +\frac{\partial}{\partial \eta}\left\{\left[1+\varepsilon_{I} g_{I}(\chi, \eta, \phi, T)\right] \frac{\partial \tilde{I}(\chi, \eta, \phi, \vartheta)}{\partial \eta}\right\} \\
& \times \frac{D_{0 I}}{\sqrt{D_{0 I} D_{0 V}}}+\frac{D_{0 I}}{\sqrt{D_{0 I} D_{0 V}}} \\
& \times \frac{\partial}{\partial \phi}\left\{\left[1+\varepsilon_{I} g_{I}(\chi, \eta, \phi, T)\right] \frac{\partial \tilde{I}(\chi, \eta, \phi, \vartheta)}{\partial \phi}\right\} \\
& -\tilde{I}(\chi, \eta, \phi, \vartheta) \omega\left[1+\varepsilon_{I, V} g_{I, V}(\chi, \eta, \phi, T)\right] \tilde{V}(\chi, \eta, \phi, \vartheta) \\
& -\Omega_{I}\left[1+\varepsilon_{I, I} g_{I, I}(\chi, \eta, \phi, T)\right] \tilde{I}^{2}(\chi, \eta, \phi, \vartheta), \\
\frac{\partial \tilde{V}(\chi, \eta, \phi, \vartheta)}{\partial \vartheta}= & \frac{D_{0 V}}{\sqrt{D_{0 I} D_{0 V}}} \frac{\partial}{\partial \chi}\left\{\left[1+\varepsilon_{V} g_{V}(\chi, \eta, \phi, T)\right] \frac{\partial \tilde{V}(\chi, \eta, \phi, \vartheta)}{\partial \chi}\right\} \\
& +\frac{\partial}{\partial \eta}\left\{\left[1+\varepsilon_{V} g_{V}(\chi, \eta, \phi, T)\right] \frac{\partial \tilde{V}(\chi, \eta, \phi, \vartheta)}{\partial \eta}\right\} \\
& \times \frac{D_{0 V}}{\sqrt{D_{0 I} D_{0 V}}}+\frac{D_{0 V}}{\sqrt{D_{0 I} D_{0 V}}} \\
&
\end{aligned}
$$




$$
\begin{gathered}
\times \frac{\partial}{\partial \phi}\left\{\left[1+\varepsilon_{V} g_{V}(\chi, \eta, \phi, T)\right] \frac{\partial \tilde{V}(\chi, \eta, \phi, \vartheta)}{\partial \phi}\right\} \\
-\widetilde{I}(\chi, \eta, \phi, \vartheta) \times \omega\left[1+\varepsilon_{I, V} g_{I, V}(\chi, \eta, \phi, T)\right] \widetilde{V}(\chi, \eta, \phi, \vartheta) \\
-\Omega_{V}\left[1+\varepsilon_{V, V} g_{V, V}(\chi, \eta, \phi, T)\right] \tilde{V}^{2}(\chi, \eta, \phi, \vartheta) \\
\left.\frac{\partial \widetilde{\rho}(\chi, \eta, \phi, \vartheta)}{\partial \chi}\right|_{\chi=0}=0,\left.\quad \frac{\partial \tilde{\rho}(\chi, \eta, \phi, \vartheta)}{\partial \chi}\right|_{\chi=1}=0,\left.\quad \frac{\partial \widetilde{\rho}(\chi, \eta, \phi, \vartheta)}{\partial \eta}\right|_{\eta=0}=0 \\
\left.\frac{\partial \tilde{\rho}(\chi, \eta, \phi, \vartheta)}{\partial \eta}\right|_{\eta=1}=0,\left.\quad \frac{\partial \widetilde{\rho}(\chi, \eta, \phi, \vartheta)}{\partial \phi}\right|_{\phi=0}=0,\left.\quad \frac{\partial \tilde{\rho}(\chi, \eta, \phi, \vartheta)}{\partial \phi}\right|_{\phi=1}=0 \\
\tilde{\rho}(\chi, \eta, \phi, \vartheta)=\frac{f_{\rho}(\chi, \eta, \phi, \vartheta)}{\rho^{*}}
\end{gathered}
$$

We determine solutions of Equations (8) with conditions (9) framework recently introduced approach [18], i.e., as the power series

$$
\widetilde{\rho}(\chi, \eta, \phi, \vartheta)=\sum_{i=0}^{\infty} \varepsilon^{i} \rho \sum_{j=0}^{\infty} \omega^{j} \sum_{k=0}^{\infty} \Omega_{\rho}^{k} \widetilde{\rho}_{i j k}(\chi, \eta, \phi, \vartheta) .
$$

Substitution of the series (10) into Equations (8) and conditions (9) gives us possibility to obtain equations for initial-order approximations of concentration of point defects $\widetilde{I}_{000}(\chi, \eta, \phi, \vartheta)$ and $\tilde{V}_{000}(\chi, \eta, \phi, \vartheta)$ and corrections for them $\tilde{I}_{i j k}(\chi, \eta, \phi, \vartheta)$ and $\tilde{V}_{i j k}(\chi, \eta, \phi, \vartheta), i \geq 1, j \geq 1, k \geq 1$. The equations are presented in the Appendix. Solutions of the equations could be obtained by standard Fourier approach [24, 25]. The solutions are presented in the Appendix.

Now we calculate distributions of concentrations of simplest complexes of point radiation defects in space and time. To determine the distributions we transform approximations of diffusion coefficients in the following form: $D_{\Phi \rho}(x, y, z, T)=D_{0 \Phi \rho}\left[1+\varepsilon_{\Phi \rho} g_{\Phi \rho}(x, y, z, T)\right]$, where 
$D_{0 \Phi \rho}$ are the average values of diffusion coefficients. In this situation the Equations (6) could be written as

$$
\begin{aligned}
\frac{\partial \Phi_{I}(x, y, z, t)}{\partial t}= & D_{0 \Phi I} \frac{\partial}{\partial x}\left\{\left[1+\varepsilon_{\Phi I} g_{\Phi I}(x, y, z, T)\right] \frac{\partial \Phi_{I}(x, y, z, t)}{\partial x}\right\} \\
& +k_{I, I}(x, y, z, T) I^{2}(x, y, z, t) \\
& +D_{0 \Phi I} \frac{\partial}{\partial y}\left\{\left[1+\varepsilon_{\Phi I} g_{\Phi I}(x, y, z, T)\right] \frac{\partial \Phi_{I}(x, y, z, t)}{\partial y}\right\} \\
& +D_{0 \Phi I} \frac{\partial}{\partial z}\left\{\left[1+\varepsilon_{\Phi I} g_{\Phi I}(x, y, z, T)\right] \frac{\partial \Phi_{I}(x, y, z, t)}{\partial z}\right\} \\
& -k_{I}(x, y, z, T) I(x, y, z, t), \\
\frac{\partial \Phi_{V}(x, y, z, t)}{\partial t}= & D_{0 \Phi V} \frac{\partial}{\partial x}\left\{\left[1+\varepsilon_{\Phi V} g_{\Phi V}(x, y, z, T)\right] \frac{\partial \Phi_{V}(x, y, z, t)}{\partial x}\right\} \\
& +k_{I, I}(x, y, z, T) I^{2}(x, y, z, t) \\
& +D_{0 \Phi V} \frac{\partial}{\partial y}\left\{\left[1+\varepsilon_{\Phi V} g_{\Phi V}(x, y, z, T)\right] \frac{\partial \Phi_{V}(x, y, z, t)}{\partial y}\right\} \\
& +D_{0 \Phi V} \frac{\partial}{\partial z}\left\{\left[1+\varepsilon_{\Phi V} g_{\Phi V}(x, y, z, T)\right] \frac{\partial \Phi_{V}(x, y, z, t)}{\partial z}\right\} \\
& -k_{I}(x, y, z, T) I(x, y, z, t) .
\end{aligned}
$$

Farther we determine solutions of above equations as the following power series:

$$
\Phi_{\rho}(x, y, z, t)=\sum_{i=0}^{\infty} \varepsilon_{\Phi \rho}^{i} \Phi_{\rho i}(x, y, z, t) .
$$

Now we used the series (11) into Equations (6) and appropriate boundary and initial conditions. The using gives the possibility to obtain equations for initial-order approximations of concentrations of complexes 
of defects $\Phi_{\rho 0}(x, y, z, t)$, corrections for them $\Phi_{\rho i}(x, y, z, t)$ (for them $i \geq 1$ ) and boundary and initial conditions for them. We remove equations and conditions to the Appendix. Solutions of the equations have been calculated by standard approaches $[24,25]$ and presented in the Appendix.

Now we calculate distribution of concentration of dopant in space and time by using the approach, which was used for analysis of radiation defects. To use the approach we consider following transformation of approximation of dopant diffusion coefficient: $D_{L}(x, y, z, T)=D_{0 L}$ $\left[1+\varepsilon_{L} g_{L}(x, y, z, T)\right]$, where $D_{0 L}$ is the average value of dopant diffusion coefficient, $0 \leq \varepsilon_{L}<1,\left|g_{L}(x, y, z, T)\right| \leq 1$. Farther we consider the solution of Equation (1) as the following series:

$$
C(x, y, z, t)=\sum_{i=0}^{\infty} \varepsilon_{L}^{i} \sum_{j=1}^{\infty} \xi^{j} C_{i j}(x, y, z, t) .
$$

Using the relation into Equation (1) and conditions (2) leads to obtaining equations for the functions $C_{i j}(x, y, z, t)(i \geq 1, j \geq 1)$, boundary and initial conditions for them. The equations are presented in the Appendix. Solutions of the equations have been calculated by standard approaches (see, for example, [24, 25]). The solutions are presented in the Appendix.

We analyzed distributions of concentrations of dopant and radiation defects in space and time analytically by using the second-order approximations on all parameters, which have been used in appropriate series. Usually the second-order approximations are enough good approximations to make qualitative analysis and to obtain quantitative results. All analytical results have been checked by numerical simulation. 


\section{Discussion}

In this section we analyzed spatio-temporal distributions of concentrations of dopants. Figures 2 show typical spatial distributions of concentrations of dopants in neighbourhood of interfaces of heterostructures. We calculate these distributions of concentrations of dopants under the following condition: value of dopant diffusion coefficient in doped area is larger, than value of dopant diffusion coefficient in nearest areas. In this situation, one can find increasing of compactness of field-effect transistors with increasing of homogeneity of distribution of concentration of dopant at one time. Changing relation between values of dopant diffusion coefficients leads to opposite result (see Figures 3).

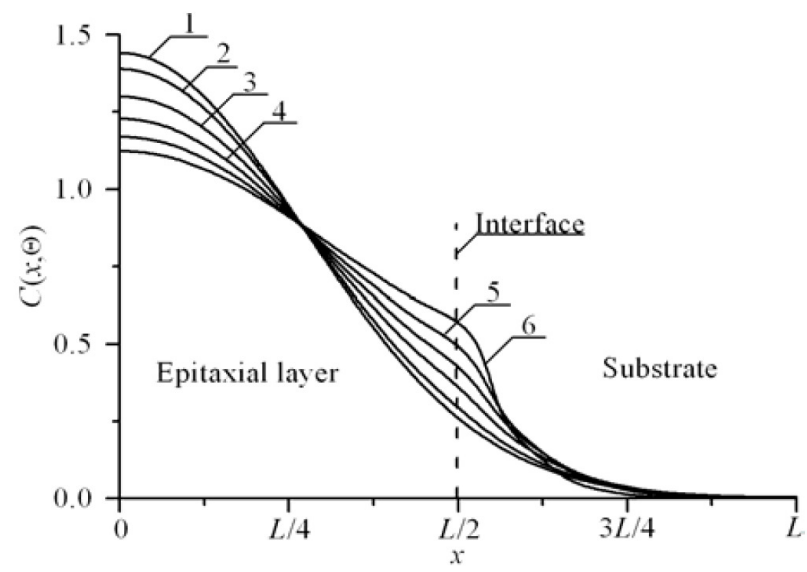

Figure 2(a). Dependences of concentration of dopant, infused in heterostructure from Figure 1, on coordinate in direction, which is perpendicular to interface between epitaxial layer substrate. Difference between values of dopant diffusion coefficient in layers of heterostructure increases with increasing of number of curves. Value of dopant diffusion coefficient in the epitaxial layer is larger, than value of dopant diffusion coefficient in the substrate. 


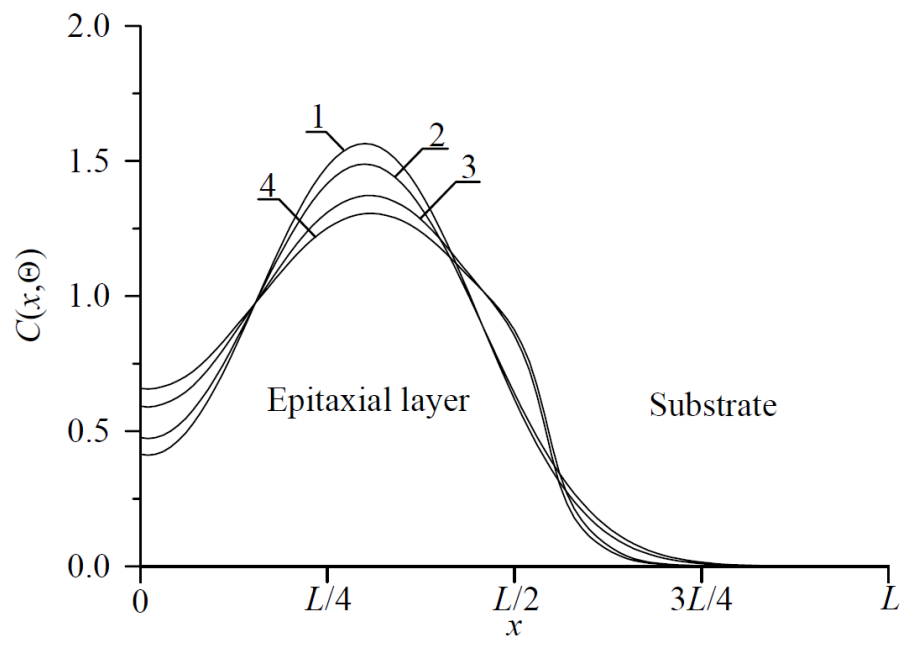

Figure 2(b). Dependences of concentration of dopant, implanted in heterostructure from Figure 1, on coordinate in direction, which is perpendicular to interface between epitaxial layer substrate. Difference between values of dopant diffusion coefficient in layers of heterostructure increases with increasing of number of curves. Value of dopant diffusion coefficient in the epitaxial layer is larger, than value of dopant diffusion coefficient in the substrate. Curve 1 corresponds to homogeneous sample and annealing time $\Theta=0.0048\left(L_{x}{ }^{2}+L_{y}{ }^{2}+L_{z}{ }^{2}\right) / D_{0}$. Curve 2 corresponds to homogeneous sample and annealing time $\Theta=0.0057\left(L_{x}{ }^{2}+L_{y}{ }^{2}+L_{z}{ }^{2}\right) / D_{0}$. Curves 3 and 4 correspond to heterostructure from Figure 1; annealing times $\Theta=0.0048\left(L_{x}{ }^{2}+L_{y}{ }^{2}+L_{z}{ }^{2}\right) / D_{0}$ and $\Theta=0.0057\left(L_{x}^{2}+L_{y}{ }^{2}+L_{z}{ }^{2}\right) / D_{0}$, respectively. 


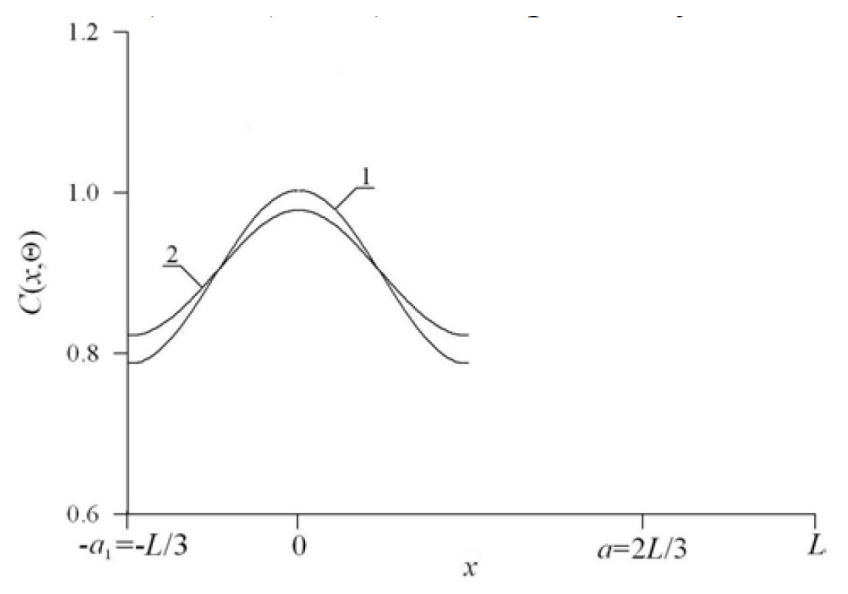

Figure 3(a). Distributions of concentration of dopant, infused in average section of epitaxial layer of heterostructure from Figure 1 in direction parallel to interface between epitaxial layer and substrate of heterostructure. Difference between values of dopant diffusion coefficients increases with increasing of number of curves. Value of dopant diffusion coefficient in this section is smaller, than value of dopant diffusion coefficient in nearest sections.

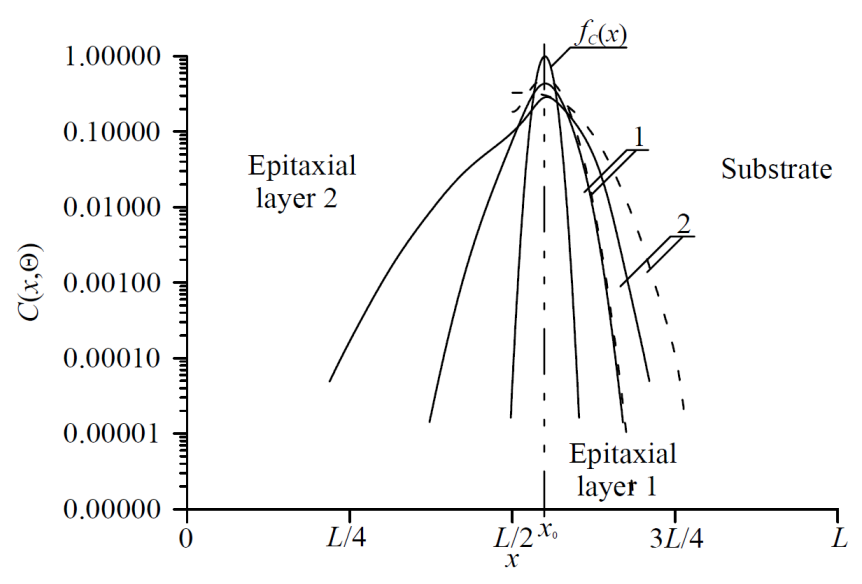

Figure 3(b). Calculated distributions of implanted dopant in epitaxial layers of heterostructure. Solid lines are spatial distributions of implanted dopant in system of two epitaxial layers. Dashed lines are spatial distributions of implanted dopant in one epitaxial layer. Annealing time increases with increasing of number of curves. 
It should be noted, that framework the considered approach one shall optimize annealing of dopant and/or radiation defects. To do the optimization we used recently introduced criterion [26-34]. The optimization based on approximation real distribution by step-wise function $\psi(x, y, z)$ (see Figures 4). Farther the required values of optimal annealing time have been calculated by minimization the following mean squared error:

$$
U=\frac{1}{L_{x} L_{y} L_{z}} \int_{0}^{L_{x}} \int_{0}^{L_{y}} \int_{0}^{L_{z}}[C(x, y, z, \Theta)-\psi(x, y, z)] d z d y d x .
$$

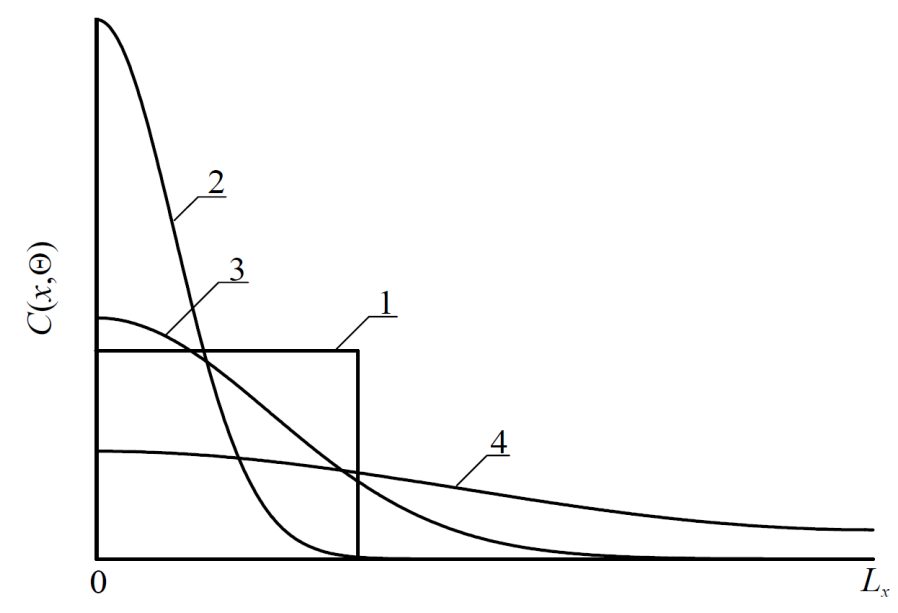

Figure 4(a). Distributions of concentration of infused dopant in depth of heterostructure from Figure 1 for different values of annealing time (curves 2-4) and idealized step-wise approximation (curve 1). Increasing of number of curve corresponds to increasing of annealing time. 


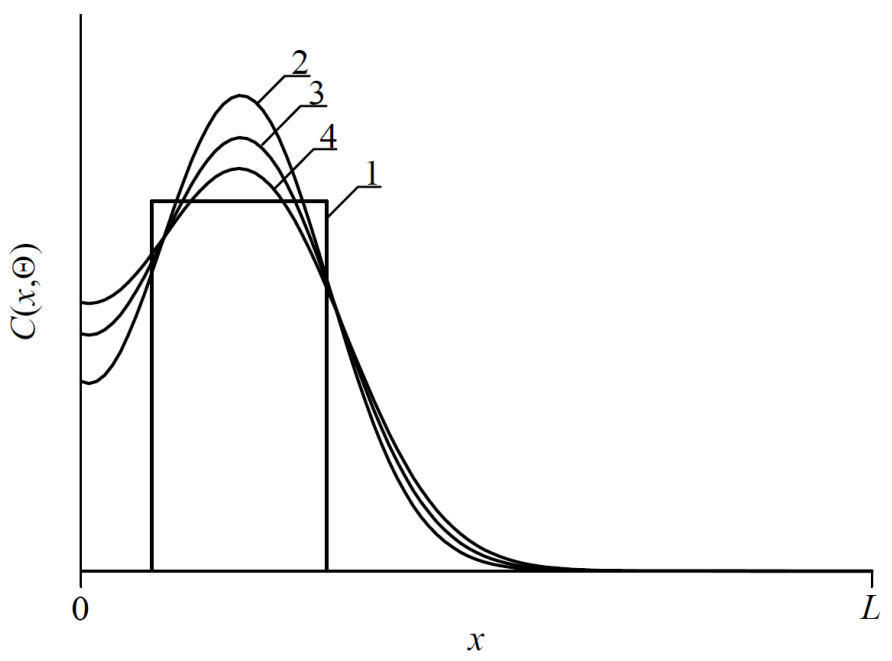

Figure 4(b). Distributions of concentration of implanted dopant in depth of heterostructure from Figure 1 for different values of annealing time (curves 2-4) and idealized step-wise approximation (curve 1). Increasing of number of curve corresponds to increasing of annealing time.

We show optimal values of annealing time as functions of parameters on Figures 5. It is known, that standard step of manufactured ion-doped structures is annealing of radiation defects. In the ideal case after finishing the annealing dopant achieves interface between layers of heterostructure. If the dopant has no enough time to achieve the interface, it is practicably to anneal the dopant additionally. The Figure 5(b) shows the described dependences of optimal values of additional annealing time for the same parameters as for Figure 5(a). Necessity to anneal radiation defects leads to smaller values of optimal annealing of implanted dopant in comparison with optimal annealing time of infused dopant. 


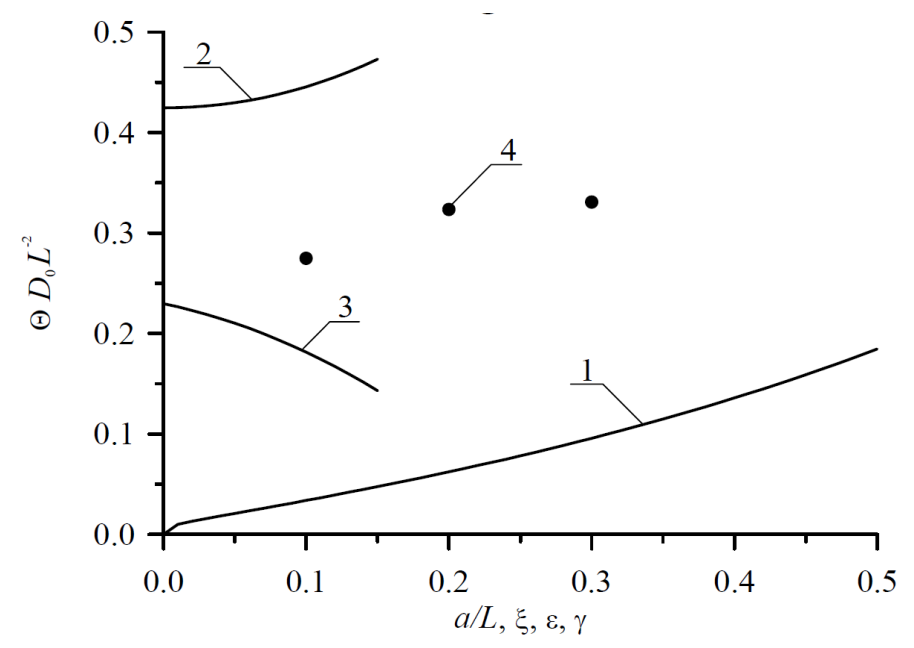

Figure 5(a). Dimensionless optimal annealing time of infused dopant as a function of several parameters. Curve 1 describes the dependence of the annealing time on the relation $a / L$ and $\xi=\gamma=0$ for equal to each other values of dopant diffusion coefficient in all parts of heterostructure. Curve 2 describes the dependence of the annealing time on value of parameter $\varepsilon$ for $a / L=1 / 2$ and $\xi=\gamma=0$. Curve 3 describes the dependence of the annealing time on value of parameter $\xi$ for $a / L=1 / 2$ and $\varepsilon=\gamma=0$. Curve 4 describes the dependence of the annealing time on value of parameter $\gamma$ for $a / L=1 / 2$ and $\varepsilon=\xi=0$. 


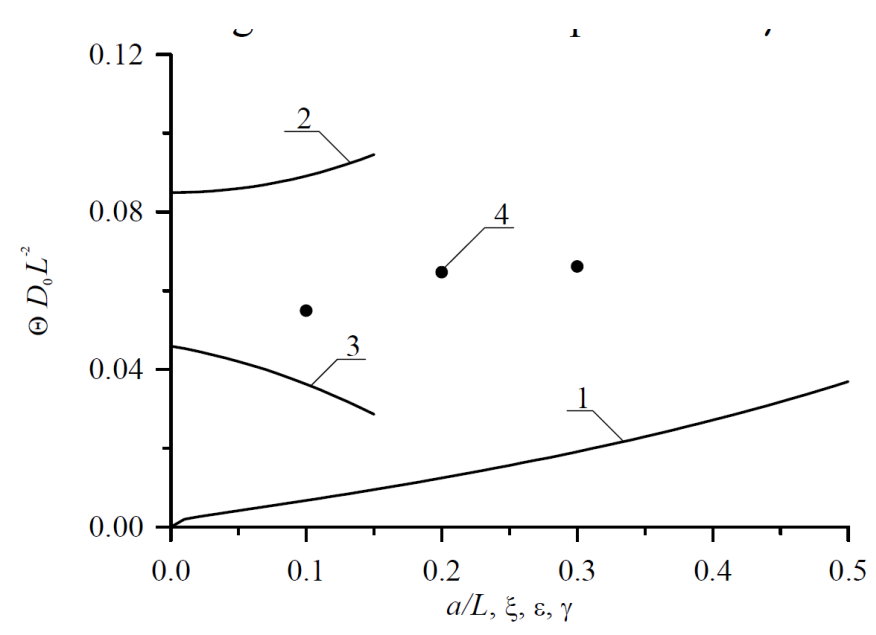

Figure 5(b). Dimensionless optimal annealing time of implanted dopant as a function of several parameters. Curve 1 describes the dependence of the annealing time on the relation $a / L$ and $\xi=\gamma=0$ for equal to each other values of dopant diffusion coefficient in all parts of heterostructure. Curve 2 describes the dependence of the annealing time on value of parameter $\varepsilon$ for $a / L=1 / 2$ and $\xi=\gamma=0$. Curve 3 describes the dependence of the annealing time on value of parameter $\xi$ for $a / L=1 / 2$ and $\varepsilon=\gamma=0$. Curve 4 describes the dependence of the annealing time on value of parameter $\gamma$ for $a / L=1 / 2$ and $\varepsilon=\xi=0$.

\section{Conclusion}

In this paper, we introduce an approach to increase integration rate of element of a circuit of a bit-cell without read-port transistors. The approach gives us possibility to decrease area of the elements with smaller increasing of the element's thickness.

\section{References}

[1] V. I. Lachin and N. S. Savelov, Electronics, Phoenix, Rostov-na-Donu, 2001.

[2] A. G. Alexenko and I. I. Shagurin, Microcircuitry, Radio and Communication, Moscow, 1990.

[3] N. A. Avaev, Yu. E. Naumov and V. T. Frolkin, Basis of Microelectronics, Radio and Communication, Moscow, 1991. 
[4] L. A. Solovyeva and P. G. Kirichenko, Organizing the memory array of multi-port register files to reduce power consumption, Russian Microelectronics 46(5) (2017), 334-342.

DOI: https://doi.org/10.1134/S1063739717050109

[5] D. Fathi, B. Forouzandeh and N. Masoumi, New enhanced noise analysis in active mixers in nanoscale technologies, Nano 4(4) (2009), 233-238.

DOI: https://doi.org/10.1142/S1793292009001708

[6] S. A. M. Chachuli, P. N. A. Fasyar, N. Soin, N. M. Karim and N. Yusop, Pareto ANOVA analysis for CMOS $0.18 \mu \mathrm{m}$ two-stage Op-amp, Materials Science in Semiconductor Processing 24 (2014), 9-14.

DOI: https://doi.org/10.1016/j.mssp.2014.02.035

[7] A. O. Ageev, A. E. Belyaev, N. S. Boltovets, V. N. Ivanov, R. V. Konakova, Ya. Ya. Kudryk, P. M. Lytvyn, V. V. Milenin and A. V. Sachenko, Au-TiB $x-n-6 H-S i C$ Schottky barrier diodes: Specific features of charge transport in rectifying and nonrectifying contacts, Semiconductors 43(7) (2009), 865-871.

DOI: https://doi.org/10.1134/S1063782609070070

[8] Z. Li, J. Waldron, T. Detchprohm, C. Wetzel, R. F. Karlicek Jr. and T. P. Chow, Monolithic integration of light-emitting diodes and power metal-oxidesemiconductor channel high-electron-mobility transistors for light-emitting power integrated circuits in GaN on sapphire substrate, Applied Physics Letters 102(19) (2013); Article 192107.

DOI: https://doi.org/10.1063/1.4807125

[9] Jung-Hui Tsai, Shao-Yen Chiu, Wen-Shiung Lour and Der-Feng Guo, Highperformance InGaP/GaAs pnp $\delta$-doped heterojunction bipolar transistor, Semiconductors 43(7) (2009), 939-942.

DOI: https://doi.org/10.1134/S1063782609070227

[10] O. V. Aleksandrov, A. O. Zakhar'in, N. A. Sobolev, E. I. Shek, M. M. Makoviichuk and E. O. Parshin, Formation of donor centers upon annealing of dysprosium-and holmium-implanted silicon, Semiconductors 32(9) (1998), 921-923.

DOI: https://doi.org/10.1134/1.1187515

[11] M. J. Kumar and T. V. Singh, Int. J. Nanoscience 7(2-3) (2008), 81-84.

[12] P. Sinsermsuksakul, K. Hartman, S. B. Kim, J. Heo, L. Sun, H. H. Park, R. Chakraborty, T. Buonassisi and R. G. Gordon, Enhancing the efficiency of SnS solar cells via band-offset engineering with a zinc oxysulfide buffer layer, Applied Physics Letters 102(5) (2013); Article 053901.

DOI: https://doi.org/10.1063/1.4789855 
[13] J. G. Reynolds, C. L. Reynolds Jr., A. Mohanta, J. F. Muth, J. E. Rowe, H. O. Everitt and D. E. Aspnes, Shallow acceptor complexes in p-type ZnO, Applied Physics Letters 102(15) (2013); Article 152114.

DOI: https://doi.org/10.1063/1.4802753

[14] K. K. Ong, K. L. Pey, P. S. Lee, A. T. S. Wee, X. C. Wang and Y. F. Chong, Dopant distribution in the recrystallization transient at the maximum melt depth induced by laser annealing, Applied Physics Letters 89(17) (2006); Article 172111.

DOI: https://doi.org/10.1063/1.2364834

[15] H. T. Wang, L. S. Tan and E. F. Chor, Pulsed laser annealing of Be-implanted GaN, Journal of Applied Physics 98(9) (2005); Article 094901.

DOI: https://doi.org/10.1063/1.2120893

[16] S. T. Sisianu, T. S. Sisianu and S. K. Railean, Shallow $p-n$ junctions formed in silicon using pulsed photon annealing, Semiconductors 36(5) (2002), 581-587.

DOI: https://doi.org/10.1134/1.1478552

[17] Yu. V. Bykov, A. G. Eremeev, N. A. Zharova, I. V. Plotnikov, K. I. Rybakov, M. N. Drozdov, Yu. N. Drozdov and V. D. Skupov, Diffusion processes in semiconductor structures during microwave annealing, Radiophysics and Quantum Electronics 46(8-9) (2003), 749-755.

DOI: https://doi.org/10.1023/B:RAQE.0000025008.97954.1c

[18] E. L. Pankratov and E. A. Bulaeva, Doping of materials during manufacture $p$ - $n$-junctions and bipolar transistors: Analytical approaches to model technological approaches and ways of optimization of distributions of dopants, Reviews in Theoretical Science 1(1) (2013), 58-82.

DOI: https://doi.org/10.1166/rits.2013.1004

[19] Yu. N. Erofeev, Pulse Devices, Higher School, Moscow, 1989.

[20] V. V. Kozlivsky, Modification of Semiconductors by Proton Beams, Nauka, Saint Petersburg, 2003.

[21] Z. Yu. Gotra, Technology of Microelectronic Devices, Radio and Communication, Moscow, 1991.

[22] V. L. Vinetskiy and G. A. Kholodar, Radiative Physics of Semiconductors, Naukova Dumka, Kiev, 1979.

[23] P. M. Fahey, P. B. Griffin and J. D. Plummer, Point defects and dopant diffusion in silicon, Reviews of Modern Physics 61(2) (1989); Article 289.

DOI: https://doi.org/10.1103/RevModPhys.61.289 
[24] A. N. Tikhonov and A. A. Samarskii, The Mathematical Physics Equations, Moscow, Nauka, 1972.

[25] H. S. Carslaw and J. C. Jaeger, Conduction of Heat in Solids, Oxford University Press, 1964.

[26] E. L. Pankratov, Dopant diffusion dynamics and optimal diffusion time as influenced by diffusion-coefficient nonuniformity, Russian Microelectronics 36(1) (2007), 33-39.

DOI: https://doi.org/10.1134/S1063739707010040

[27] E. L. Pankratov, Redistribution of a dopant during annealing of radiation defects in a multilayer structure by laser scans for production of an implanted-junction rectifier, International Journal of Nanoscience 7(4-5) (2008), 187-197.

DOI: https://doi.org/10.1142/S0219581X08005328

[28] E. L. Pankratov, On approach to optimize manufacturing of bipolar heterotransistors framework circuit of an operational amplifier to increase their integration rate: influence mismatch-induced stress, Journal of Computational and Theoretical Nanoscience 14(10) (2017), 4885-4899.

\section{DOI: https://doi.org/10.1166/jctn.2017.6899}

[29] E. L. Pankratov, On optimization of manufacturing of two-phase logic circuit based on heterostructures to increase density of their elements: Influence of miss-match induced stress, Advanced Science, Engineering and Medicine 9(9) (2017), 787-801.

DOI: https://doi.org/10.1166/asem.2017.2043

[30] E. L. Pankratov and E. A. Bulaeva, An approach to decrease dimensions of fieldeffect transistors, Universal Journal of Materials Science 1(1) (2013), 6-11.

DOI: https://doi.org/10.13189/ujms.2013.010102

[31] E. L. Pankratov and E. A. Bulaeva, An approach to manufacture a heterobipolar transistors in thin film structures: On the method of optimization, International Journal of Micro-Nano Scale Transport 4(1) (2014), 17-32.

DOI: http://dx.doi.org/10.1260/1759-3093.4.1-2.17

[32] E. L. Pankratov and E. A. Bulaeva, Application of native inhomogeneities to increase compactness of vertical field-effect transistors, Journal of Nanoengineering and Nanomanufacturing 2(3) (2012), 275-280.

DOI: https://doi.org/10.1166/jnan.2012.1084

[33] E. L. Pankratov and E. A. Bulaeva, An approach to increase the integration rate of planar drift heterobipolar transistors, Materials Science in Semiconductor Processing 34 (2015), 260-268.

DOI: https://doi.org/10.1016/j.mssp.2015.02.054 


\section{Appendix}

Equations for the functions $\widetilde{I}_{i j k}(\chi, \eta, \phi, \vartheta)$ and $\tilde{V}_{i j k}(\chi, \eta, \phi, \vartheta), i \geq 0$, $j \geq 0, k \geq 0$ and conditions for them

$$
\begin{aligned}
& \frac{\partial \widetilde{I}_{000}(\chi, \eta, \phi, \vartheta)}{\partial \vartheta}=\sqrt{\frac{D_{0 I}}{D_{0 V}}}\left[\frac{\partial^{2} \widetilde{I}_{000}(\chi, \eta, \phi, \vartheta)}{\partial \chi^{2}}\right. \\
& \left.+\frac{\partial^{2} \widetilde{I}_{000}(\chi, \eta, \phi, \vartheta)}{\partial \eta^{2}}+\frac{\partial^{2} \widetilde{I}_{000}(\chi, \eta, \phi, \vartheta)}{\partial \phi^{2}}\right], \\
& \frac{\partial \tilde{V}_{000}(\chi, \eta, \phi, \vartheta)}{\partial \vartheta}=\sqrt{\frac{D_{0 V}}{D_{0 I}}}\left[\frac{\partial^{2} \tilde{V}_{000}(\chi, \eta, \phi, \vartheta)}{\partial \chi^{2}}\right. \\
& \left.+\frac{\partial^{2} \tilde{V}_{000}(\chi, \eta, \phi, \vartheta)}{\partial \eta^{2}}+\frac{\partial^{2} \tilde{V}_{000}(\chi, \eta, \phi, \vartheta)}{\partial \phi^{2}}\right], \\
& \frac{\partial \tilde{I}_{i 00}(\chi, \eta, \phi, \vartheta)}{\partial \vartheta}=\sqrt{\frac{D_{0 I}}{D_{0 V}}}\left[\frac{\partial^{2} \widetilde{I}_{i 00}(\chi, \eta, \phi, \vartheta)}{\partial \chi^{2}}\right. \\
& \left.+\frac{\partial^{2} \widetilde{I}_{i 00}(\chi, \eta, \phi, \vartheta)}{\partial \eta^{2}}+\frac{\partial^{2} \widetilde{I}_{i 00}(\chi, \eta, \phi, \vartheta)}{\partial \phi^{2}}\right] \\
& +\sqrt{\frac{D_{0 I}}{D_{0 V}}}\left\{\frac{\partial}{\partial \chi}\left[g_{I}(\chi, \eta, \phi, T) \frac{\partial \widetilde{I}_{i-100}(\chi, \eta, \phi, \vartheta)}{\partial \chi}\right]\right. \\
& +\frac{\partial}{\partial \eta}\left[g_{I}(\chi, \eta, \phi, T) \frac{\partial \tilde{I}_{i-100}(\chi, \eta, \phi, \vartheta)}{\partial \eta}\right] \\
& \left.+\frac{\partial}{\partial \phi}\left[g_{I}(\chi, \eta, \phi, T) \frac{\partial \widetilde{I}_{i-100}(\chi, \eta, \phi, \vartheta)}{\partial \phi}\right]\right\}, i \geq 1
\end{aligned}
$$




$$
\begin{aligned}
& \frac{\partial \tilde{V}_{i 00}(\chi, \eta, \phi, \vartheta)}{\partial \vartheta}=\sqrt{\frac{D_{0 V}}{D_{0 I}}}\left[\frac{\partial^{2} \tilde{V}_{i 00}(\chi, \eta, \phi, \vartheta)}{\partial \chi^{2}}\right. \\
& \left.+\frac{\partial^{2} \widetilde{V}_{i 00}(\chi, \eta, \phi, \vartheta)}{\partial \eta^{2}}+\frac{\partial^{2} \widetilde{V}_{i 00}(\chi, \eta, \phi, \vartheta)}{\partial \phi^{2}}\right] \\
& +\sqrt{\frac{D_{0 V}}{D_{0 I}}}\left\{\frac{\partial}{\partial \chi}\left[g_{V}(\chi, \eta, \phi, T) \frac{\partial \tilde{V}_{i-100}(\chi, \eta, \phi, \vartheta)}{\partial \chi}\right]\right. \\
& +\frac{\partial}{\partial \eta}\left[g_{V}(\chi, \eta, \phi, T) \frac{\partial \widetilde{V}_{i-100}(\chi, \eta, \phi, \vartheta)}{\partial \eta}\right] \\
& \left.+\frac{\partial}{\partial \phi}\left[g_{V}(\chi, \eta, \phi, T) \frac{\partial \widetilde{V}_{i-100}(\chi, \eta, \phi, \vartheta)}{\partial \phi}\right]\right\}, i \geq 1, \\
& \frac{\partial \tilde{I}_{010}(\chi, \eta, \phi, \vartheta)}{\partial \vartheta}=\sqrt{\frac{D_{0 I}}{D_{0 V}}}\left[\frac{\partial^{2} \widetilde{I}_{010}(\chi, \eta, \phi, \vartheta)}{\partial \chi^{2}}\right. \\
& \left.+\frac{\partial^{2} \widetilde{I}_{010}(\chi, \eta, \phi, \vartheta)}{\partial \eta^{2}}+\frac{\partial^{2} \widetilde{I}_{010}(\chi, \eta, \phi, \vartheta)}{\partial \phi^{2}}\right] \\
& -\left[1+\varepsilon_{I, V} g_{I, V}(\chi, \eta, \phi, T)\right] \\
& \times \widetilde{I}_{000}(\chi, \eta, \phi, \vartheta) \widetilde{V}_{000}(\chi, \eta, \phi, \vartheta) \\
& \frac{\partial \tilde{V}_{010}(\chi, \eta, \phi, \vartheta)}{\partial \vartheta}=\sqrt{\frac{D_{0 V}}{D_{0 I}}}\left[\frac{\partial^{2} \widetilde{V}_{010}(\chi, \eta, \phi, \vartheta)}{\partial \chi^{2}}\right. \\
& \left.+\frac{\partial^{2} \widetilde{V}_{010}(\chi, \eta, \phi, \vartheta)}{\partial \eta^{2}}+\frac{\partial^{2} \widetilde{V}_{010}(\chi, \eta, \phi, \vartheta)}{\partial \phi^{2}}\right] \\
& -\left[1+\varepsilon_{I, V} g_{I, V}(\chi, \eta, \phi, T)\right] \\
& \times \widetilde{I}_{000}(\chi, \eta, \phi, \vartheta) \widetilde{V}_{000}(\chi, \eta, \phi, \vartheta)
\end{aligned}
$$




$$
\begin{aligned}
& \frac{\partial \tilde{I}_{020}(\chi, \eta, \phi, \vartheta)}{\partial \vartheta}=\sqrt{\frac{D_{0 I}}{D_{0 V}}}\left[\frac{\partial^{2} \widetilde{I}_{020}(\chi, \eta, \phi, \vartheta)}{\partial \chi^{2}}\right. \\
& \left.+\frac{\partial^{2} \widetilde{I}_{020}(\chi, \eta, \phi, \vartheta)}{\partial \eta^{2}}+\frac{\partial^{2} \widetilde{I}_{020}(\chi, \eta, \phi, \vartheta)}{\partial \phi^{2}}\right] \\
& -\left[1+\varepsilon_{I, V} g_{I, V}(\chi, \eta, \phi, T)\right] \\
& \times\left[\widetilde{I}_{010}(\chi, \eta, \phi, \vartheta) \widetilde{V}_{000}(\chi, \eta, \phi, \vartheta)\right. \\
& \left.+\widetilde{I}_{000}(\chi, \eta, \phi, \vartheta) \tilde{V}_{010}(\chi, \eta, \phi, \vartheta)\right], \\
& \frac{\partial \tilde{V}_{020}(\chi, \eta, \phi, \vartheta)}{\partial \vartheta}=\sqrt{\frac{D_{0 V}}{D_{0 I}}}\left[\frac{\partial^{2} \tilde{V}_{020}(\chi, \eta, \phi, \vartheta)}{\partial \chi^{2}}\right. \\
& \left.+\frac{\partial^{2} \widetilde{V}_{020}(\chi, \eta, \phi, \vartheta)}{\partial \eta^{2}}+\frac{\partial^{2} \widetilde{V}_{020}(\chi, \eta, \phi, \vartheta)}{\partial \phi^{2}}\right] \\
& -\left[1+\varepsilon_{I, V} g_{I, V}(\chi, \eta, \phi, T)\right] \\
& \times\left[\widetilde{I}_{010}(\chi, \eta, \phi, \vartheta) \widetilde{V}_{000}(\chi, \eta, \phi, \vartheta)\right. \\
& \left.+\tilde{I}_{000}(\chi, \eta, \phi, \vartheta) \widetilde{V}_{010}(\chi, \eta, \phi, \vartheta)\right], \\
& \frac{\partial \tilde{I}_{001}(\chi, \eta, \phi, \vartheta)}{\partial \vartheta}=\sqrt{\frac{D_{0 I}}{D_{0 V}}}\left[\frac{\partial^{2} \widetilde{I}_{001}(\chi, \eta, \phi, \vartheta)}{\partial \chi^{2}}\right. \\
& \left.+\frac{\partial^{2} \widetilde{I}_{001}(\chi, \eta, \phi, \vartheta)}{\partial \eta^{2}}+\frac{\partial^{2} \widetilde{I}_{001}(\chi, \eta, \phi, \vartheta)}{\partial \phi^{2}}\right] \\
& -\left[1+\varepsilon_{I, I} g_{I, I}(\chi, \eta, \phi, T)\right] \tilde{I}_{000}^{2}(\chi, \eta, \phi, \vartheta), \\
& \frac{\partial \tilde{V}_{001}(\chi, \eta, \phi, \vartheta)}{\partial \vartheta}=\sqrt{\frac{D_{0 V}}{D_{0 I}}}\left[\frac{\partial^{2} \widetilde{V}_{001}(\chi, \eta, \phi, \vartheta)}{\partial \chi^{2}}\right. \\
& \left.+\frac{\partial^{2} \widetilde{V}_{001}(\chi, \eta, \phi, \vartheta)}{\partial \eta^{2}}+\frac{\partial^{2} \widetilde{V}_{001}(\chi, \eta, \phi, \vartheta)}{\partial \phi^{2}}\right] \\
& -\left[1+\varepsilon_{I, I} g_{I, I}(\chi, \eta, \phi, T)\right] \tilde{V}_{000}^{2}(\chi, \eta, \phi, \vartheta),
\end{aligned}
$$




$$
\begin{aligned}
& \frac{\partial \widetilde{I}_{110}(\chi, \eta, \phi, \vartheta)}{\partial \vartheta}=\sqrt{\frac{D_{0 I}}{D_{0 V}}}\left[\frac{\partial^{2} \widetilde{I}_{110}(\chi, \eta, \phi, \vartheta)}{\partial \chi^{2}}\right. \\
& \left.+\frac{\partial^{2} \widetilde{I}_{110}(\chi, \eta, \phi, \vartheta)}{\partial \eta^{2}}+\frac{\partial^{2} \widetilde{I}_{110}(\chi, \eta, \phi, \vartheta)}{\partial \phi^{2}}\right] \\
& +\sqrt{\frac{D_{0 I}}{D_{0 V}}}\left\{\frac{\partial}{\partial \chi}\left[g_{I}(\chi, \eta, \phi, T) \frac{\partial \tilde{I}_{010}(\chi, \eta, \phi, \vartheta)}{\partial \chi}\right]\right. \\
& +\frac{\partial}{\partial \eta}\left[g_{I}(\chi, \eta, \phi, T) \frac{\partial \widetilde{I}_{010}(\chi, \eta, \phi, \vartheta)}{\partial \eta}\right] \\
& \left.+\frac{\partial}{\partial \phi}\left[g_{I}(\chi, \eta, \phi, T) \frac{\partial \tilde{I}_{010}(\chi, \eta, \phi, \vartheta)}{\partial \phi}\right]\right\} \\
& -\left[\widetilde{I}_{100}(\chi, \eta, \phi, \vartheta) \tilde{V}_{000}(\chi, \eta, \phi, \vartheta)\right. \\
& \left.+\widetilde{I}_{000}(\chi, \eta, \phi, \vartheta) \widetilde{V}_{100}(\chi, \eta, \phi, \vartheta)\right] \\
& \times\left[1+\varepsilon_{I, I} g_{I, I}(\chi, \eta, \phi, T)\right], \\
& \frac{\partial \widetilde{V}_{110}(\chi, \eta, \phi, \vartheta)}{\partial \vartheta}=\sqrt{\frac{D_{0 V}}{D_{0 I}}}\left[\frac{\partial^{2} \widetilde{V}_{110}(\chi, \eta, \phi, \vartheta)}{\partial \chi^{2}}\right. \\
& \left.+\frac{\partial^{2} \tilde{V}_{110}(\chi, \eta, \phi, \vartheta)}{\partial \eta^{2}}+\frac{\partial^{2} \tilde{V}_{110}(\chi, \eta, \phi, \vartheta)}{\partial \phi^{2}}\right] \\
& +\sqrt{\frac{D_{0 V}}{D_{0 I}}}\left\{\frac{\partial}{\partial \chi}\left[g_{V}(\chi, \eta, \phi, T) \frac{\partial \tilde{V}_{010}(\chi, \eta, \phi, \vartheta)}{\partial \chi}\right]\right. \\
& +\frac{\partial}{\partial \eta}\left[g_{V}(\chi, \eta, \phi, T) \frac{\partial \tilde{V}_{010}(\chi, \eta, \phi, \vartheta)}{\partial \eta}\right] \\
& \left.+\frac{\partial}{\partial \phi}\left[g_{V}(\chi, \eta, \phi, T) \frac{\partial \tilde{V}_{010}(\chi, \eta, \phi, \vartheta)}{\partial \phi}\right]\right\}
\end{aligned}
$$




$$
\begin{aligned}
& -\left[1+\varepsilon_{V, V} g_{V, V}(\chi, \eta, \phi, T)\right] \\
& \times\left[\widetilde{V}_{100}(\chi, \eta, \phi, \vartheta) \widetilde{I}_{000}(\chi, \eta, \phi, \vartheta)\right. \\
& \left.+\widetilde{V}_{000}(\chi, \eta, \phi, \vartheta) \widetilde{I}_{100}(\chi, \eta, \phi, \vartheta)\right] \\
& \frac{\partial \tilde{I}_{002}(\chi, \eta, \phi, \vartheta)}{\partial \vartheta}=\sqrt{\frac{D_{0 I}}{D_{0 V}}}\left[\frac{\partial^{2} \widetilde{I}_{002}(\chi, \eta, \phi, \vartheta)}{\partial \chi^{2}}\right. \\
& \left.+\frac{\partial^{2} \widetilde{I}_{002}(\chi, \eta, \phi, \vartheta)}{\partial \eta^{2}}+\frac{\partial^{2} \widetilde{I}_{002}(\chi, \eta, \phi, \vartheta)}{\partial \phi^{2}}\right] \\
& -\left[1+\varepsilon_{I, I} g_{I, I}(\chi, \eta, \phi, T)\right] \\
& \times \widetilde{I}_{001}(\chi, \eta, \phi, \vartheta) \widetilde{I}_{000}(\chi, \eta, \phi, \vartheta) \\
& \frac{\partial \tilde{V}_{002}(\chi, \eta, \phi, \vartheta)}{\partial \vartheta}=\sqrt{\frac{D_{0 V}}{D_{0 I}}}\left[\frac{\partial^{2} \widetilde{V}_{002}(\chi, \eta, \phi, \vartheta)}{\partial \chi^{2}}\right. \\
& \left.+\frac{\partial^{2} \widetilde{V}_{002}(\chi, \eta, \phi, \vartheta)}{\partial \eta^{2}}+\frac{\partial^{2} \widetilde{V}_{002}(\chi, \eta, \phi, \vartheta)}{\partial \phi^{2}}\right] \\
& -\left[1+\varepsilon_{V, V} g_{V, V}(\chi, \eta, \phi, E)\right] \\
& \times \tilde{V}_{001}(\chi, \eta, \phi, \vartheta) \tilde{V}_{000}(\chi, \eta, \phi, \vartheta), \\
& \frac{\partial \widetilde{I}_{101}(\chi, \eta, \phi, \vartheta)}{\partial \vartheta}=\sqrt{\frac{D_{0 I}}{D_{0 V}}}\left[\frac{\partial^{2} \widetilde{I}_{101}(\chi, \eta, \phi, \vartheta)}{\partial \chi^{2}}\right. \\
& \left.+\frac{\partial^{2} \widetilde{I}_{101}(\chi, \eta, \phi, \vartheta)}{\partial \eta^{2}}+\frac{\partial^{2} \widetilde{I}_{101}(\chi, \eta, \phi, \vartheta)}{\partial \phi^{2}}\right] \\
& +\sqrt{\frac{D_{0 I}}{D_{0 V}}}\left\{\frac{\partial}{\partial \chi}\left[g_{I}(\chi, \eta, \phi, T) \frac{\partial \tilde{I}_{001}(\chi, \eta, \phi, \vartheta)}{\partial \chi}\right]\right. \\
& +\frac{\partial}{\partial \eta}\left[g_{I}(\chi, \eta, \phi, T) \frac{\partial \widetilde{I}_{001}(\chi, \eta, \phi, \vartheta)}{\partial \eta}\right]
\end{aligned}
$$




$$
\begin{aligned}
& \left.+\frac{\partial}{\partial \phi}\left[g_{I}(\chi, \eta, \phi, T) \frac{\partial \widetilde{I}_{001}(\chi, \eta, \phi, \vartheta)}{\partial \phi}\right]\right\} \\
& -\left[1+\varepsilon_{I} g_{I}(\chi, \eta, \phi, T)\right] \\
& \times \widetilde{I}_{100}(\chi, \eta, \phi, \vartheta) \widetilde{V}_{000}(\chi, \eta, \phi, \vartheta) \\
& \frac{\partial \widetilde{V}_{101}(\chi, \eta, \phi, \vartheta)}{\partial \vartheta}=\sqrt{\frac{D_{0 V}}{D_{0 I}}}\left[\frac{\partial^{2} \widetilde{V}_{101}(\chi, \eta, \phi, \vartheta)}{\partial \chi^{2}}\right. \\
& \left.+\frac{\partial^{2} \widetilde{V}_{101}(\chi, \eta, \phi, \vartheta)}{\partial \eta^{2}}+\frac{\partial^{2} \widetilde{V}_{101}(\chi, \eta, \phi, \vartheta)}{\partial \phi^{2}}\right] \\
& +\sqrt{\frac{D_{0 V}}{D_{0 I}}}\left\{\frac{\partial}{\partial \chi}\left[g_{V}(\chi, \eta, \phi, T) \frac{\partial \tilde{V}_{001}(\chi, \eta, \phi, \vartheta)}{\partial \chi}\right]\right. \\
& +\frac{\partial}{\partial \eta}\left[g_{V}(\chi, \eta, \phi, T) \frac{\partial \tilde{V}_{001}(\chi, \eta, \phi, \vartheta)}{\partial \eta}\right] \\
& \left.+\frac{\partial}{\partial \phi}\left[g_{V}(\chi, \eta, \phi, T) \frac{\partial \widetilde{V}_{001}(\chi, \eta, \phi, \vartheta)}{\partial \phi}\right]\right\} \\
& -\left[1+\varepsilon_{V} g_{V}(\chi, \eta, \phi, T)\right] \\
& \times \widetilde{I}_{000}(\chi, \eta, \phi, \vartheta) \widetilde{V}_{100}(\chi, \eta, \phi, \vartheta) \\
& \frac{\partial \widetilde{I}_{011}(\chi, \eta, \phi, \vartheta)}{\partial \vartheta}=\sqrt{\frac{D_{0 I}}{D_{0 V}}}\left[\frac{\partial^{2} \widetilde{I}_{011}(\chi, \eta, \phi, \vartheta)}{\partial \chi^{2}}\right. \\
& \left.+\frac{\partial^{2} \widetilde{I}_{011}(\chi, \eta, \phi, \vartheta)}{\partial \eta^{2}}+\frac{\partial^{2} \widetilde{I}_{011}(\chi, \eta, \phi, \vartheta)}{\partial \phi^{2}}\right] \\
& -\widetilde{I}_{010}(\chi, \eta, \phi, \vartheta)\left[1+\varepsilon_{I, I} g_{I, I}(\chi, \eta, \phi, T)\right] \\
& \times \widetilde{I}_{000}(\chi, \eta, \phi, \vartheta)-\left[1+\varepsilon_{I, V} g_{I, V}(\chi, \eta, \phi, T)\right] \\
& \times \widetilde{I}_{001}(\chi, \eta, \phi, \vartheta) \widetilde{V}_{000}(\chi, \eta, \phi, \vartheta),
\end{aligned}
$$




$$
\begin{aligned}
& \frac{\partial \tilde{V}_{011}(\chi, \eta, \phi, \vartheta)}{\partial \vartheta}=\sqrt{\frac{D_{0 V}}{D_{0 I}}}\left[\frac{\partial^{2} \widetilde{V}_{011}(\chi, \eta, \phi, \vartheta)}{\partial \chi^{2}}\right. \\
& \left.+\frac{\partial^{2} \widetilde{V}_{011}(\chi, \eta, \phi, \vartheta)}{\partial \eta^{2}}+\frac{\partial^{2} \widetilde{V}_{011}(\chi, \eta, \phi, \vartheta)}{\partial \phi^{2}}\right] \\
& -\tilde{V}_{010}(\chi, \eta, \phi, \vartheta)\left[1+\varepsilon_{V, V} g_{V, V}(\chi, \eta, \phi, T)\right] \\
& \times \tilde{V}_{000}(\chi, \eta, \phi, \vartheta)-\left[1+\varepsilon_{I, V} g_{I, V}(\chi, \eta, \phi, t)\right] \\
& \times \widetilde{I}_{000}(\chi, \eta, \phi, \vartheta) \widetilde{V}_{001}(\chi, \eta, \phi, \vartheta) \\
& \left.\frac{\partial \tilde{\rho}_{i j k}(\chi, \eta, \phi, \vartheta)}{\partial \chi}\right|_{x=0}=0,\left.\quad \frac{\partial \tilde{\rho}_{i j k}(\chi, \eta, \phi, \vartheta)}{\partial \chi}\right|_{x=1}=0,\left.\quad \frac{\partial \tilde{\rho}_{i j k}(\chi, \eta, \phi, \vartheta)}{\partial \eta}\right|_{\eta=0}=0, \\
& \left.\frac{\partial \tilde{\rho}_{i j k}(\chi, \eta, \phi, \vartheta)}{\partial \eta}\right|_{\eta=1}=0,\left.\quad \frac{\partial \tilde{\rho}_{i j k}(\chi, \eta, \phi, \vartheta)}{\partial \phi}\right|_{\phi=0}=0,\left.\quad \frac{\partial \tilde{\rho}_{i j k}(\chi, \eta, \phi, \vartheta)}{\partial \phi}\right|_{\phi=1}=0 \\
& i \geq 0, j \geq 0, k \geq 0 \text {; }
\end{aligned}
$$$$
\tilde{\rho}_{000}(\chi, \eta, \phi, 0)=f_{\rho}(\chi, \eta, \phi) / \rho^{*}, \tilde{\rho}_{i j k}(\chi, \eta, \phi, 0)=0(i \geq 1, j \geq 1, k \geq 1) .
$$

Solutions of the above equations could be written as

$$
\widetilde{\rho}_{000}(\chi, \eta, \phi, \vartheta)=\frac{1}{L}+\frac{2}{L} \sum_{n=1}^{\infty} F_{n \rho} c(\chi) c(\eta) c(\phi) e_{n \rho}(\vartheta)
$$

where

$$
\begin{aligned}
& F_{n \rho}=\frac{1}{\rho^{*}} \int_{0}^{1} \cos (\pi n u) \int_{0}^{1} \cos (\pi n \nu) \int_{0}^{1} \cos (\pi n w) F_{n \rho}(u, \nu, w) d w d \nu d u, c_{n}(\chi)=\cos (\pi n \chi) \\
& e_{n I}(\vartheta)=\exp \left(-\pi^{2} n^{2} \vartheta \sqrt{D_{0 V} / D_{0 I}}\right), \text { and } e_{n V}(\vartheta)=\exp \left(-\pi^{2} n^{2} \vartheta \sqrt{D_{0 I} / D_{0 V}}\right)
\end{aligned}
$$




$$
\begin{aligned}
& \tilde{I}_{i 00}(\chi, \eta, \phi, \vartheta)=-2 \pi \sqrt{\frac{D_{0 I}}{D_{0 V}}} \sum_{n=1}^{\infty} n c_{n}(\chi) c(\eta) c(\phi) e_{n I}(\vartheta) \int_{0}^{\vartheta} e_{n I}(-\tau) \int_{0}^{1} s_{n}(u) \int_{0}^{1} c_{n}(\nu) \\
& \times \int_{0}^{1} \frac{\partial \tilde{I}_{i-100}(u, \nu, w, \tau)}{\partial u} c_{n}(w) g_{I}(u, \nu, w, T) d w d \nu d u d \tau \\
& -2 \pi \sqrt{\frac{D_{0 I}}{D_{0 V}}} \sum_{n=1}^{\infty} n c_{n}(\chi) c(\eta) c(\phi) e_{n I}(\vartheta) \int_{0}^{\vartheta} e_{n I}(-\tau) \int_{0}^{1} c_{n}(u) \int_{0}^{1} s_{n}(\nu) \\
& \times \int_{0}^{1} c_{n}(w) g_{I}(u, \nu, w, T) \frac{\partial \widetilde{I}_{i-100}(u, \nu, w, \tau)}{\partial \nu} d w d \nu d u d \tau \\
& -2 \pi \sqrt{\frac{D_{0 I}}{D_{0 V}}} \sum_{n=1}^{\infty} n c_{n}(\chi) c(\eta) c(\phi) e_{n I}(\vartheta) \int_{0}^{\vartheta} e_{n I}(-\tau) \int_{0}^{1} c_{n}(u) \int_{0}^{1} c_{n}(\nu) \\
& \times \int_{0}^{1} s_{n}(w) g_{I}(u, \nu, w, T) \frac{\partial \widetilde{I}_{i-100}(u, \nu, w, \tau)}{\partial w} d w d \nu d u d \tau, i \geq 1 \\
& \tilde{V}_{i 00}(\chi, \eta, \phi, \vartheta)=-2 \pi \sqrt{\frac{D_{0 V}}{D_{0 I}}} \sum_{n=1}^{\infty} n c_{n}(\chi) c(\eta) c(\phi) e_{n V}(\vartheta) \int_{0}^{\vartheta} e_{n V}(-\tau) \int_{0}^{1} s_{n}(u) \int_{0}^{1} c_{n}(\nu) \\
& \times \int_{0}^{1} g_{V}(u, \nu, w, T) c_{n}(w) \frac{\partial \tilde{V}_{i-100}(u, \nu, w, \tau)}{\partial u} d w d \nu d u d \tau \\
& -2 \pi \sqrt{\frac{D_{0 V}}{D_{0 I}}} \sum_{n=1}^{\infty} n c_{n}(\chi) c(\eta) c(\phi) e_{n V}(\vartheta) \int_{0}^{\vartheta} e_{n V}(-\tau) \int_{0}^{1} c_{n}(u) \int_{0}^{1} s_{n}(\nu) \\
& \times \int_{0}^{1} c_{n}(w) g_{V}(u, \nu, w, T) \frac{\partial \tilde{V}_{i-100}(u, \nu, w, \tau)}{\partial \nu} d w d \nu d u d \tau
\end{aligned}
$$




$$
\begin{aligned}
& -2 \pi \sqrt{\frac{D_{0 V}}{D_{0 I}}} \sum_{n=1}^{\infty} n c_{n}(\chi) c(\eta) c(\phi) e_{n V}(\vartheta) \int_{0}^{\vartheta} e_{n V}(-\tau) \int_{0}^{1} c_{n}(u) \int_{0}^{1} c_{n}(\nu) \\
& \times \int_{0}^{1} s_{n}(w) g_{V}(u, \nu, w, T) \frac{\partial \tilde{V}_{i-100}(u, \nu, w, \tau)}{\partial w} d w d \nu d u d \tau, i \geq 1,
\end{aligned}
$$

where $s_{n}(\chi)=\sin (\pi n \chi)$;

$$
\begin{aligned}
\tilde{\rho}_{010}(\chi, \eta, \phi, \vartheta)= & -2 \sum_{n=1}^{\infty} c_{n}(\chi) c_{n}(\eta) c_{n}(\phi) e_{n \rho}(\vartheta) \int_{0}^{\vartheta} e_{n \rho}(-\tau) \int_{0}^{1} c_{n}(u) \int_{0}^{1} c_{n}(\nu) \int_{0}^{1} c_{n}(w) \\
& \times\left[1+\varepsilon_{I, V} g_{I, V}(u, \nu, w, T)\right] \tilde{I}_{000}(u, \nu, w, \tau) \\
& \times \tilde{V}_{000}(u, \nu, w, \tau) d w d \nu d u d \tau, \\
\tilde{\rho}_{020}(\chi, \eta, \phi, \vartheta)=- & 2 \sqrt{\frac{D_{0 I}}{D_{0 V}} \sum_{n=1}^{\infty} c_{n}(\chi) c_{n}(\eta) c_{n}(\phi) e_{n \rho}(\vartheta) \int_{0}^{\vartheta} e_{n \rho}(-\tau) \int_{0}^{1} c_{n}(u) \int_{0}^{1} c_{n}(\nu)} \\
& \times \int_{0}^{1} c_{n}(w)\left[1+\varepsilon \varepsilon_{I, V} g I, V(u, \nu, w, T)\right]\left[\tilde{I}_{010}(u, \nu, w, \tau)\right. \\
& \times \tilde{V}_{000}(u, \nu, w, \tau)+\tilde{I}_{000}(u, \nu, w, \tau) \\
& \left.\times \tilde{V}_{010}(u, \nu, w, \tau)\right] d w d \nu d u d \tau, \\
& \times\left[1+\varepsilon_{\rho, \rho} g_{\rho, \rho}(u, \nu, w, T)\right] \tilde{\rho}_{000}^{2}(u, \nu, w, \tau) d w d \nu d u d \tau, \\
\tilde{\rho}_{001}(\chi, \eta, \phi, \vartheta)= & -2 \sum_{n=1}^{\infty} c_{n}(\chi) c_{n}(\eta) c_{n}(\phi) e_{n \rho}(\vartheta) \int_{0}^{\vartheta} e_{n \rho}(-\tau) \int_{0}^{1} c_{n}(u) \int_{0}^{1} c_{n}(\nu) \int_{0}^{1} c_{n}(w) \\
&
\end{aligned}
$$




$$
\begin{aligned}
& \tilde{\rho}_{002}(\chi, \eta, \phi, \vartheta)=-2 \sum_{n=1}^{\infty} c_{n}(\chi) c_{n}(\eta) c_{n}(\phi) e_{n \rho}(\vartheta) \int_{0}^{\vartheta} e_{n \rho}(-\tau) \int_{0}^{1} c_{n}(u) \int_{0}^{1} c_{n}(\nu) \int_{0}^{1} c_{n}(w) \\
& \times\left[1+\varepsilon_{\rho, \rho} g_{\rho, \rho}(u, \nu, w, T)\right] \widetilde{\rho}_{001}(u, \nu, w, \tau) \\
& \times \widetilde{\rho}_{000}(u, \nu, w, \tau) d w d \nu d u d \tau, \\
& \tilde{I}_{110}(\chi, \eta, \phi, \vartheta)=-2 \pi \sqrt{\frac{D_{0 I}}{D_{0 V}}} \sum_{n=1}^{\infty} n c_{n}(\chi) c_{n}(\eta) c_{n}(\phi) e_{n I}(\vartheta) \int_{0}^{\vartheta} e_{n I}(-\tau) \int_{0}^{1} s_{n}(u) \int_{0}^{1} c_{n}(\nu) \\
& \times \int_{0}^{1} c_{n}(u) g_{I}(u, \nu, w, T) \frac{\partial \tilde{I}_{i-100}(u, \nu, w, \tau)}{\partial u} d w d \nu d u d \tau \\
& -2 \pi \sqrt{\frac{D_{0 I}}{D_{0 V}}} \sum_{n=1}^{\infty} n c_{n}(\chi) c_{n}(\eta) c_{n}(\phi) e_{n I}(\vartheta) \int_{0}^{\vartheta} e_{n I}(-\tau) \int_{0}^{1} c_{n}(u) \int_{0}^{1} s_{n}(\nu) \\
& \times \int_{0}^{1} c_{n}(u) g_{I}(u, \nu, w, T) \frac{\partial \tilde{I}_{i-100}(u, \nu, w, \tau)}{\partial \nu} d w d \nu d u d \tau \\
& -2 \pi \sqrt{\frac{D_{0 I}}{D_{0 V}}} \sum_{n=1}^{\infty} n e_{n I}(\vartheta) \int_{0}^{\vartheta} e_{n I}(-\tau) \int_{0}^{1} c_{n}(u) \int_{0}^{1} c_{n}(\nu) \\
& \times \int_{0}^{1} s_{n}(u) g_{I}(u, \nu, w, T) \frac{\partial \widetilde{I}_{i-100}(u, \nu, w, \tau)}{\partial w} d w d \nu d u d \tau \\
& \times c_{n}(\chi) c_{n}(\eta) c_{n}(\phi)-2 \sum_{n=1}^{\infty} c_{n}(\chi) e_{n I}(\vartheta) c_{n}(\eta) c_{n}(\phi) \\
& \times \int_{0}^{\vartheta} e_{n I}(-\tau) \int_{0}^{1} c_{n}(u) \int_{0}^{1} c_{n}(\nu) \int_{0}^{1} c_{n}(w)\left[1+\varepsilon_{I, V} g_{I, V}(u, \nu, w, T)\right] \\
& \times\left[\tilde{I}_{100}(u, \nu, w, \tau) \tilde{V}_{000}(u, \nu, w, \tau)+\widetilde{I}_{000}(u, \nu, w, \tau)\right. \\
& \left.\times \tilde{V}_{100}(u, \nu, w, \tau)\right] d w d \nu d u d \tau,
\end{aligned}
$$




$$
\begin{aligned}
& \tilde{V}_{110}(\chi, \eta, \phi, \vartheta)=-2 \pi \sqrt{\frac{D_{0 V}}{D_{0 I}}} \sum_{n=1}^{\infty} n c_{n}(\chi) c_{n}(\eta) c_{n}(\phi) e_{n V}(\vartheta) \int_{0}^{\vartheta} e_{n V}(-\tau) \int_{0}^{1} s_{n}(u) \\
& \times \int_{0}^{1} c_{n}(\nu) \int_{0}^{1} c_{n}(u) g_{V}(u, \nu, w, T) \frac{\partial \widetilde{V}_{i-100}(u, \nu, w, \tau)}{\partial u} d w d \nu d u d \tau \\
& -2 \pi \sqrt{\frac{D_{0 V}}{D_{0 I}}} \sum_{n=1}^{\infty} n c_{n}(\chi) c_{n}(\eta) c_{n}(\phi) e_{n V}(\vartheta) \int_{0}^{\vartheta} e_{n V}(-\tau) \int_{0}^{1} c_{n}(u) \\
& \times \int_{0}^{1} s_{n}(\nu) \int_{0}^{1} c_{n}(u) g_{V}(u, \nu, w, T) \frac{\partial \tilde{V}_{i-100}(u, \nu, w, \tau)}{\partial \nu} d w d \nu d u d \tau \\
& -2 \pi \sqrt{\frac{D_{0 V}}{D_{0 I}}} \times \sum_{n=1}^{\infty} n e_{n V}(\vartheta) \int_{0}^{\vartheta} e_{n V}(-\tau) \int_{0}^{1} c_{n}(u) \int_{0}^{1} c_{n}(\nu) \\
& \times \int_{0}^{1} s_{n}(u) g_{V}(u, \nu, w, T) \frac{\partial \tilde{V}_{i-100}(u, \nu, w, \tau)}{\partial w} d w d \nu d u d \tau \\
& \times c_{n}(\chi) c_{n}(\eta) c_{n}(\phi)-2 \sum_{n=1}^{\infty} c_{n}(\chi) e_{n V}(\vartheta) c_{n}(\eta) c_{n}(\phi) \int_{0}^{\vartheta} e_{n V}(-\tau) \\
& \times \int_{0}^{1} c_{n}(u) \int_{0}^{1} c_{n}(\nu) \int_{0}^{1}\left[1+\varepsilon_{I, V} g_{I, V}(u, \nu, w, T)\right] c_{n}(w) \\
& \times\left[\widetilde{I}_{100}(u, \nu, w, \tau) \widetilde{V}_{000}(u, \nu, w, \tau)+\widetilde{I}_{000}(u, \nu, w, \tau)\right. \\
& \left.\times \tilde{V}_{100}(u, \nu, w, \tau)\right] d w d \nu d u d \tau,
\end{aligned}
$$




$$
\begin{aligned}
I_{101}(\chi, \eta, \phi, \vartheta)= & -2 \pi \sqrt{\frac{D_{0 I}}{D_{0 V}}} \sum_{n=1}^{\infty} n c_{n}(\chi) c_{n}(\eta) c_{n}(\phi) e_{n I}(\vartheta) \int_{0}^{\vartheta} e_{n I}(-\tau) \int_{0}^{1} s_{n}(u) \int_{0}^{1} c_{n}(\nu) \\
& \times \int_{0}^{1} g_{I}(u, \nu, w, T) c_{n}(w) \frac{\partial \widetilde{I}_{001}(u, \nu, w, \tau)}{\partial u} d w d \nu d u d \tau \\
& -2 \pi \sqrt{\frac{D_{0 I}}{D_{0 V}}} \sum_{n=1}^{\infty} n c_{n}(\chi) c_{n}(\eta) c_{n}(\phi) e_{n I}(\vartheta) \int_{0}^{1} s_{n}(\nu) \\
& \times \int_{0}^{1} c_{n}(w) g_{I}(u, \nu, w, T) \frac{\partial \tilde{I}_{001}(u, \nu, w, \tau)}{\partial \nu} d w d \nu d u d \tau \\
& -2 \pi \sqrt{\frac{D_{0 I}}{D_{0 V}} \sum_{n=1}^{\infty} n e_{n I}(\vartheta) c_{n}(\chi) c_{n}(\eta) c_{n}(\phi) \int_{0}^{\vartheta} e_{n I}(-\tau) \int_{0}^{1} c_{n}(u)} \\
& \times \widetilde{V}_{000}(u, \nu, w, \tau) d w d \nu d u d \tau, \\
& \times \int_{0}^{1} c_{n}(\nu) \int_{0}^{1} s_{n}(w) g_{I}(u, \nu, w, T) \frac{\partial \tilde{I}_{001}(u, \nu, w, \tau)}{\partial w} d w d \nu d u d \tau \\
& -2 \sum_{n=1}^{\infty} c_{n}(\chi) c_{n}(\eta) c_{n}(\phi) e_{n I}(\vartheta) \int_{0}^{\vartheta} e_{n I}(-\tau) \int_{0}^{1} c_{n}(u) \int_{0}^{1} c_{n}(\nu) \int_{0}^{1} c_{n}(w) \\
& {\left[\varepsilon_{I, V} g_{I, V}(u, \nu, w, T)\right] \tilde{I}_{100}(u, \nu, w, \tau) } \\
& \\
& \\
&
\end{aligned}
$$




$$
\begin{aligned}
& \tilde{V}_{101}(\chi, \eta, \phi, \vartheta)=-2 \pi \sqrt{\frac{D_{0 V}}{D_{0 I}}} \sum_{n=1}^{\infty} n c_{n}(\chi) c_{n}(\eta) c_{n}(\phi) e_{n V}(\vartheta) \int_{0}^{\vartheta} e_{n V}(-\tau) \int_{0}^{1} s_{n}(u) \\
& \times \int_{0}^{1} c_{n}(\nu) \int_{0}^{1} g_{V}(u, \nu, w, T) c_{n}(w) \frac{\partial \tilde{V}_{001}(u, \nu, w, \tau)}{\partial u} d w d \nu d u d \tau \\
& -2 \pi \sqrt{\frac{D_{0 V}}{D_{0 I}}} \sum_{n=1}^{\infty} n c_{n}(\chi) c_{n}(\eta) c_{n}(\phi) e_{n V}(\vartheta) \int_{0}^{\vartheta} e_{n V}(-\tau) \int_{0}^{1} c_{n}(u) \\
& \times \int_{0}^{1} s_{n}(\nu) \int_{0}^{1} c_{n}(w) g_{I}(u, \nu, w, T) \frac{\partial \widetilde{V}_{001}(u, \nu, w, \tau)}{\partial \nu} d w d \nu d u d \tau \\
& -2 \pi \sqrt{\frac{D_{0 I}}{D_{0 V}}} \sum_{n=1}^{\infty} n e_{n I}(\vartheta) c_{n}(\chi) c_{n}(\eta) c_{n}(\phi) \int_{0}^{\vartheta} e_{n V}(-\tau) \int_{0}^{1} c_{n}(u) \\
& \times \int_{0}^{1} c_{n}(\nu) \int_{0}^{1} s_{n}(w) g_{V}(u, \nu, w, T) \frac{\partial \tilde{V}_{001}(u, \nu, w, \tau)}{\partial w} d w d \nu d u d \tau \\
& -2 \sum_{n=1}^{\infty} c_{n}(\chi) c_{n}(\eta) c_{n}(\phi) e_{n V}(\vartheta) \int_{0}^{\vartheta} e_{n V}(-\tau) \int_{0}^{1} c_{n}(u) \int_{0}^{1} c_{n}(\nu) \\
& \times \int_{0}^{1} c_{n}(w)\left[1+\varepsilon_{I, V} g_{I, V}(u, \nu, w, T)\right] \\
& \times \widetilde{I}_{100}(u, \nu, w, \tau) \widetilde{V}_{000}(u, \nu, w, \tau) d w d \nu d u d \tau, \\
& \tilde{I}_{011}(\chi, \eta, \phi, \vartheta)=-2 \sum_{n=1}^{\infty} c_{n}(\chi) c_{n}(\eta) c_{n}(\phi) e_{n I}(\vartheta) \int_{0}^{\vartheta} e_{n I}(-\tau) \int_{0}^{1} c_{n}(u) \int_{0}^{1} c_{n}(\nu) \int_{0}^{1} c_{n}(w) \\
& \times\left\{\widetilde{I}_{000}(u, \nu, w, \tau)\left[1+\varepsilon_{I, I} g_{I, I}(u, \nu, w, T)\right]\right. \\
& \times \tilde{I}_{010}(u, \nu, w, \tau)+\left[1+\varepsilon_{I, V} g_{I, V}(u, \nu, w, T)\right] \\
& \left.\times \widetilde{I}_{001}(u, \nu, w, \tau) \widetilde{V}_{000}(u, \nu, w, \tau)\right\} d w d \nu d u d \tau,
\end{aligned}
$$




$$
\begin{aligned}
\tilde{V}_{011}(\chi, \eta, \phi, \vartheta)= & -2 \sum_{n=1}^{\infty} c_{n}(\chi) c_{n}(\eta) c_{n}(\phi) e_{n V}(\vartheta) \int_{0}^{\vartheta} e_{n V}(-\tau) \int_{0}^{1} c_{n}(u) \int_{0}^{1} c_{n}(\nu) \int_{0}^{1} c_{n}(w) \\
& \times\left\{\widetilde{I}_{000}(u, \nu, w, \tau) \times\left[1+\varepsilon_{I, I} g_{I, I}(u, \nu, w, T)\right]\right. \\
& \times \widetilde{I}_{010}(u, \nu, w, \tau)+\left[1+\varepsilon_{I, V} g_{I, V}(u, \nu, w, T)\right] \\
& \left.\times \widetilde{I}_{001}(u, \nu, w, \tau) \widetilde{V}_{000}(u, \nu, w, \tau)\right\} d w d \nu d u d \tau .
\end{aligned}
$$

Equations for functions $\Phi_{\rho i}(x, y, z, t), i \geq 0$ to describe concentrations of simplest complexes of radiation defects.

$$
\begin{aligned}
\frac{\partial \Phi_{I 0}(x, y, z, t)}{\partial t}= & D_{0 \Phi I}\left[\frac{\partial^{2} \Phi_{I 0}(x, y, z, t)}{\partial x^{2}}\right. \\
& \left.+\frac{\partial^{2} \Phi_{I 0}(x, y, z, t)}{\partial y^{2}}+\frac{\partial^{2} \Phi_{I 0}(x, y, z, t)}{\partial z^{2}}\right] \\
& +k_{I, I}(x, y, z, T) I^{2}(x, y, z, t) \\
& -k_{I}(x, y, z, T) I(x, y, z, t), \\
\frac{\partial \Phi_{V 0}(x, y, z, t)}{\partial t}= & D_{0 \Phi V}\left[\frac{\partial^{2} \Phi_{V 0}(x, y, z, t)}{\partial x^{2}}\right. \\
& \left.+\frac{\partial^{2} \Phi_{V 0}(x, y, z, t)}{\partial y^{2}}+\frac{\partial^{2} \Phi_{V 0}(x, y, z, t)}{\partial z^{2}}\right] \\
& +k_{V, V}(x, y, z, T) V^{2}(x, y, z, t) \\
& -k_{V}(x, y, z, T) V(x, y, z, t),
\end{aligned}
$$




$$
\begin{aligned}
\frac{\partial \Phi_{I i}(x, y, z, t)}{\partial t}= & D_{0 \Phi I}\left[\frac{\partial^{2} \Phi_{I i}(x, y, z, t)}{\partial x^{2}}\right. \\
& \left.+\frac{\partial^{2} \Phi_{I i}(x, y, z, t)}{\partial y^{2}}+\frac{\partial^{2} \Phi_{I i}(x, y, z, t)}{\partial z^{2}}\right] \\
& +D_{0 \Phi I}\left\{\frac{\partial}{\partial x}\left[g_{\Phi I}(x, y, z, T) \frac{\partial \Phi_{I i-1}(x, y, z, t)}{\partial x}\right]\right. \\
& +\frac{\partial}{\partial y}\left[g_{\Phi I}(x, y, z, T) \frac{\partial \Phi_{I i-1}(x, y, z, t)}{\partial y}\right] \\
& \left.+\frac{\partial}{\partial z}\left[g_{\Phi I}(x, y, z, T) \frac{\partial \Phi_{I i-1}(x, y, z, t)}{\partial z}\right]\right\}, i \geq 1, \\
\frac{\partial \Phi_{V i}(x, y, z, t)}{\partial t}= & D_{0 \Phi V}\left[\frac{\partial^{2} \Phi_{V i}(x, y, z, t)}{\partial x^{2}}\right] \\
& \left.+\frac{\partial}{\partial z}\left[g_{\Phi V}(x, y, z, T) \frac{\partial \Phi_{V i-1}(x, y, z, t)}{\partial z}\right]\right\}, i \geq 1 . \\
& +\frac{\partial}{\partial y}\left[g_{\Phi V}(x, y, z, T) \frac{\partial \Phi_{V i-1}(x, y, z, t)}{\partial y}\right] \\
& +D_{0 \Phi V}\left\{\frac{\partial}{\partial x}\left[g_{\Phi V}(x, y, z, T) \frac{\partial \Phi_{V i-1}(x, y, z, t)}{\partial x}\right]\right. \\
& \\
& \\
& \\
& \\
&
\end{aligned}
$$

Boundary and initial conditions for the functions takes the form

$$
\begin{gathered}
\left.\frac{\partial \Phi_{\rho i}(x, y, z, t)}{\partial x}\right|_{x=0}=0,\left.\frac{\partial \Phi_{\rho i}(x, y, z, t)}{\partial x}\right|_{x=L_{x}}=0,\left.\frac{\partial \Phi_{\rho i}(x, y, z, t)}{\partial y}\right|_{y=0}=0 \\
\left.\frac{\partial \Phi_{\rho i}(x, y, z, t)}{\partial y}\right|_{y=L_{y}}=0,\left.\frac{\partial \Phi_{\rho i}(x, y, z, t)}{\partial z}\right|_{z=0}=0,\left.\frac{\partial \Phi_{\rho i}(x, y, z, t)}{\partial z}\right|_{z=L_{z}}=0 \\
i \geq 0 ; \Phi_{\rho 0}(x, y, z, 0)=f_{\Phi \rho}(x, y, z), \Phi_{\rho i}(x, y, z, 0)=0, i \geq 1 .
\end{gathered}
$$


Solutions of the above equations could be written as

$$
\begin{aligned}
\Phi_{\rho 0}(x, y, z, t)= & \frac{1}{L_{x} L_{y} L_{z}}+\frac{2}{L_{x} L_{y} L_{z}} \sum_{n=1}^{\infty} F_{n \Phi_{\rho}} c_{n}(x) c_{n}(y) c_{n}(z) e_{n \Phi_{\rho}}(t) \\
& +\frac{2}{L} \sum_{n=1}^{\infty} n c_{n}(x) c_{n}(y) c_{n}(z) e_{\Phi_{\rho} n}(t) \\
& \times \int_{0}^{t} e_{\Phi_{\rho} n}(-\tau) \int_{0}^{L_{x}} c_{n}(u) \int_{0}^{L_{y}} c_{n}(\nu) \int_{0}^{L_{z}} c_{n}(w)\left[k_{I, I}(u, \nu, w, T)\right. \\
& \left.\times I^{2}(u, \nu, w, \tau)-k_{I}(u, \nu, w, T) I(u, \nu, w, \tau)\right] d w d \nu d u d \tau,
\end{aligned}
$$

where

$$
\begin{aligned}
& F_{n \Phi_{\rho}}=\int_{0}^{L_{x}} c_{n}(u) \int_{0}^{L_{y}} c_{n}(\nu) \int_{0}^{L_{z}} c_{n}(w) f_{\Phi_{\rho}}(u, \nu, w) d w d \nu d u, e_{n \Phi_{\rho}}(t)=\exp \left[-\pi^{2} n^{2}\right. \\
& \left.D_{0 \Phi_{\rho}} t\left(L_{x}^{-2}+L_{y}^{-2}+L_{z}^{-2}\right)\right], c_{n}(x)=\cos \left(\pi n x / L_{x}\right) \text {; } \\
& \Phi_{\rho i}(x, y, z, t)=-\frac{2 \pi}{L_{x}^{2} L_{y} L_{z}} \sum_{n=1}^{\infty} n c_{n}(x) c_{n}(y) c_{n}(z) e_{\Phi_{\rho} n}(t) \int_{0}^{t} e_{\Phi_{\rho} n}(-\tau) \int_{0}^{L_{x}} s_{n}(u) \\
& \times \int_{0}^{L_{y}} c_{n}(\nu) \int_{0}^{L_{z}} g_{\Phi_{\rho}}(u, \nu, w, T) c_{n}(w) \frac{\partial \Phi_{I_{\rho} i-1}(u, \nu, w, \tau)}{\partial u} d w d \nu d u d \tau \\
& -\frac{2 \pi}{L_{x} L_{y}^{2} L_{z}} \sum_{n=1}^{\infty} n c_{n}(x) c_{n}(y) c_{n}(z) e_{\Phi_{\rho} n}(t) \int_{0}^{t} e_{\Phi_{\rho} n}(-\tau) \\
& \times \int_{0}^{L_{x}} c_{n}(u) \int_{0}^{L_{y}} s_{n}(\nu) \int_{0}^{L_{z}} c_{n}(w) g_{\Phi_{\rho}}(u, \nu, w, T) \\
& \times \frac{\partial \Phi_{I_{\rho} i-1}(u, \nu, w, \tau)}{\partial \nu} d w d \nu d u d \tau-\frac{2 \pi}{L_{x} L_{y} L_{z}^{2}} \sum_{n=1}^{\infty} n e_{\Phi_{\rho} n}(t)
\end{aligned}
$$




$$
\begin{aligned}
& \times \int_{0}^{t} e_{\Phi_{\rho} n}(-\tau) \int_{0}^{L_{x}} c_{n}(u) \int_{0}^{L_{y}} c_{n}(\nu) \int_{0}^{L_{z}} s_{n}(w) \frac{\partial \Phi_{I_{\rho} i-1}(u, \nu, w, \tau)}{\partial w} \\
& \times g_{\Phi_{\rho}}(u, \nu, w, T) d w d \nu d u d \tau \times c_{n}(x) c_{n}(y) c_{n}(z), i \geq 1
\end{aligned}
$$

where $s_{n}(x)=\sin \left(\pi n x / L_{x}\right)$.

Equations for the functions $C_{i j}(x, y, z, t)(i \geq 0, j \geq 0)$, boundary and initial conditions could be written as

$$
\begin{aligned}
\frac{\partial C_{00}(x, y, z, t)}{\partial t}= & D_{0 L} \frac{\partial^{2} C_{00}(x, y, z, t)}{\partial x^{2}} \\
& +D_{0 L} \frac{\partial^{2} C_{00}(x, y, z, t)}{\partial y^{2}}+D_{0 L} \frac{\partial^{2} C_{00}(x, y, z, t)}{\partial z^{2}}, \\
\frac{\partial C_{i 0}(x, y, z, t)}{\partial t}= & D_{0 L}\left[\frac{\partial^{2} C_{i 0}(x, y, z, t)}{\partial x^{2}}\right. \\
& \left.+\frac{\partial^{2} C_{i 0}(x, y, z, t)}{\partial y^{2}}+\frac{\partial^{2} C_{i 0}(x, y, z, t)}{\partial z^{2}}\right] \\
& +D_{0 L} \frac{\partial}{\partial x}\left[g_{L}(x, y, z, T) \frac{\partial C_{i-10}(x, y, z, t)}{\partial x}\right] \\
& +D_{0 L} \frac{\partial}{\partial y}\left[g_{L}(x, y, z, T) \frac{\partial C_{i-10}(x, y, z, t)}{\partial y}\right] \\
& +D_{0 L} \frac{\partial}{\partial z}\left[g_{L}(x, y, z, T) \frac{\partial C_{i-10}(x, y, z, t)}{\partial z}\right], i \geq 1,
\end{aligned}
$$




$$
\begin{aligned}
& \frac{\partial C_{01}(x, y, z, t)}{\partial t}=D_{0 L} \frac{\partial^{2} C_{01}(x, y, z, t)}{\partial x^{2}} \\
& +D_{0 L} \frac{\partial^{2} C_{01}(x, y, z, t)}{\partial y^{2}}+D_{0 L} \frac{\partial^{2} C_{01}(x, y, z, t)}{\partial z^{2}} \\
& +D_{0 L} \frac{\partial}{\partial x}\left[\frac{C_{00}^{\gamma}(x, y, z, t)}{P^{\gamma}(x, y, z, T)} \frac{\partial C_{00}(x, y, z, t)}{\partial x}\right] \\
& +D_{0 L} \frac{\partial}{\partial y}\left[\frac{C_{00}^{\gamma}(x, y, z, t)}{P^{\gamma}(x, y, z, T)} \frac{\partial C_{00}(x, y, z, t)}{\partial y}\right] \\
& +D_{0 L} \frac{\partial}{\partial z}\left[\frac{C_{00}^{\gamma}(x, y, z, t)}{P^{\gamma}(x, y, z, T)} \frac{\partial C_{00}(x, y, z, t)}{\partial z}\right], \\
& \frac{\partial C_{02}(x, y, z, t)}{\partial t}=D_{0 L} \frac{\partial^{2} C_{02}(x, y, z, t)}{\partial x^{2}} \\
& +D_{0 L} \frac{\partial^{2} C_{02}(x, y, z, t)}{\partial y^{2}}+D_{0 L} \frac{\partial^{2} C_{02}(x, y, z, t)}{\partial z^{2}} \\
& +D_{0 L}\left\{\frac{\partial}{\partial x}\left[C_{01}(x, y, z, t) \frac{C_{00}^{\gamma-1}(x, y, z, t)}{P^{\gamma}(x, y, z, T)} \frac{\partial C_{00}(x, y, z, t)}{\partial x}\right]\right. \\
& +\frac{\partial}{\partial y}\left[C_{01}(x, y, z, t) \frac{C_{00}^{\gamma-1}(x, y, z, t)}{P^{\gamma}(x, y, z, T)} \frac{\partial C_{00}(x, y, z, t)}{\partial y}\right] \\
& \left.+\frac{\partial}{\partial z}\left[C_{01}(x, y, z, t) \frac{C_{00}^{\gamma-1}(x, y, z, t)}{P^{\gamma}(x, y, z, T)} \frac{\partial C_{00}(x, y, z, t)}{\partial z}\right]\right\} \\
& +D_{0 L}\left\{\frac{\partial}{\partial x}\left[\frac{C_{00}^{\gamma}(x, y, z, t)}{P^{\gamma}(x, y, z, T)} \frac{\partial C_{01}(x, y, z, t)}{\partial x}\right]\right.
\end{aligned}
$$




$$
\begin{aligned}
& +\frac{\partial}{\partial y}\left[\frac{C_{00}^{\gamma}(x, y, z, t)}{P^{\gamma}(x, y, z, T)} \frac{\partial C_{01}(x, y, z, t)}{\partial y}\right] \\
& \left.+\frac{\partial}{\partial z}\left[\frac{C_{00}^{\gamma}(x, y, z, t)}{P^{\gamma}(x, y, z, T)} \frac{\partial C_{01}(x, y, z, t)}{\partial z}\right]\right\}, \\
& \frac{\partial C_{11}(x, y, z, t)}{\partial t}=D_{0 L} \frac{\partial^{2} C_{11}(x, y, z, t)}{\partial x^{2}} \\
& +D_{0 L} \frac{\partial^{2} C_{11}(x, y, z, t)}{\partial y^{2}}+D_{0 L} \frac{\partial^{2} C_{11}(x, y, z, t)}{\partial z^{2}} \\
& +D_{0 L}\left\{\frac{\partial}{\partial x}\left[C_{10}(x, y, z, t) \frac{C_{00}^{\gamma-1}(x, y, z, t)}{P^{\gamma}(x, y, z, T)} \frac{\partial C_{00}(x, y, z, t)}{\partial x}\right]\right. \\
& +\frac{\partial}{\partial y}\left[C_{10}(x, y, z, t) \frac{C_{00}^{\gamma-1}(x, y, z, t)}{P^{\gamma}(x, y, z, T)} \frac{\partial C_{00}(x, y, z, t)}{\partial y}\right] \\
& \left.+\frac{\partial}{\partial z}\left[C_{10}(x, y, z, t) \frac{C_{00}^{\gamma-1}(x, y, z, t)}{P^{\gamma}(x, y, z, T)} \frac{\partial C_{00}(x, y, z, t)}{\partial z}\right]\right\} \\
& +D_{0 L}\left\{\frac{\partial}{\partial x}\left[\frac{C_{00}^{\gamma}(x, y, z, t)}{P^{\gamma}(x, y, z, T)} \frac{\partial C_{10}(x, y, z, t)}{\partial x}\right]\right. \\
& +\frac{\partial}{\partial y}\left[\frac{C_{00}^{\gamma}(x, y, z, t)}{P^{\gamma}(x, y, z, T)} \frac{\partial C_{10}(x, y, z, t)}{\partial y}\right] \\
& +\frac{\partial}{\partial z}\left[\frac{C_{00}^{\gamma}(x, y, z, t)}{P^{\gamma}(x, y, z, T)} \frac{\partial C_{10}(x, y, z, t)}{\partial z}\right] \\
& +D_{0 L}\left\{\frac{\partial}{\partial x}\left[g_{L}(x, y, z, T) \frac{\partial C_{01}(x, y, z, t)}{\partial x}\right]\right. \\
& +\frac{\partial}{\partial y}\left[g_{L}(x, y, z, T) \frac{\partial C_{01}(x, y, z, t)}{\partial y}\right] \\
& \left.+\frac{\partial}{\partial z}\left[g_{L}(x, y, z, T) \frac{\partial C_{01}(x, y, z, t)}{\partial z}\right]\right\},
\end{aligned}
$$




$$
\begin{gathered}
\left.\frac{\partial C_{i j}(x, y, z, t)}{\partial x}\right|_{x=0}=0,\left.\frac{\partial C_{i j}(x, y, z, t)}{\partial x}\right|_{x=L_{x}}=0,\left.\frac{\partial C_{i j}(x, y, z, t)}{\partial y}\right|_{y=0}=0, \\
\left.\frac{\partial C_{i j}(x, y, z, t)}{\partial y}\right|_{y=L_{y}}=0,\left.\frac{\partial C_{i j}(x, y, z, t)}{\partial z}\right|_{z=0}=0,\left.\frac{\partial C_{i j}(x, y, z, t)}{\partial z}\right|_{z=L_{z}}=0, \\
i \geq 0, j \geq 0 ; \\
C_{00}(x, y, z, 0)=f_{C}(x, y, z), C_{i j}(x, y, z, 0)=0, i \geq 1, j \geq 1 .
\end{gathered}
$$

Functions $C_{i j}(x, y, z, t)(i \geq 0, j \geq 0)$ could be approximated by the following series during solutions of the above equations:

$$
C_{00}(x, y, z, t)=\frac{F_{0 C}}{L_{x} L_{y} L_{z}}+\frac{2}{L_{x} L_{y} L_{z}} \sum_{n=1}^{\infty} F_{n C} c_{n}(x) c_{n}(y) c_{n}(z) e_{n C}(t) .
$$

Here $e_{n C}(t)=\exp \left[-\pi^{2} n^{2} D_{0 C} t\left(\frac{1}{L_{x}^{2}}+\frac{1}{L_{y}^{2}}+\frac{1}{L_{z}^{2}}\right)\right]$,

$$
\begin{aligned}
& F_{n C}=\int_{0}^{L_{x}} c_{n}(u) \int_{0}^{L_{y}} c_{n}(\nu) \int_{0}^{L_{z}} f_{C}(u, \nu, w) c_{n}(w) d w d \nu d u \\
& C_{i 0}(x, y, z, t)=-\frac{2 \pi}{L_{x}^{2} L_{y} L_{z}} \sum_{n=1}^{\infty} n F_{n C} c_{n}(x) c_{n}(y) c_{n}(z) e_{n C}(t) \int_{0}^{t} e_{n C}(-\tau) \int_{0}^{L_{x}} s_{n}(u) \\
& \times \int_{0}^{L_{y}} c_{n}(\nu) \int_{0}^{L_{z}} g_{L}(u, \nu, w, T) c_{n}(w) \frac{\partial C_{i-10}(u, \nu, w, \tau)}{\partial u} d w d \nu d u d \tau \\
&-\frac{2 \pi}{L_{x} L_{y}^{2} L_{z}} \sum_{n=1}^{\infty} n F_{n C} c_{n}(x) c_{n}(y) c_{n}(z) e_{n C}(t) \int_{0}^{t} e_{n C}(-\tau) \int_{0}^{L_{x}} c_{n}(u) \\
& \times \int_{0}^{L_{y}} s_{n}(\nu) \int_{0}^{L_{z}} c_{n}(\nu) g_{L}(u, \nu, w, T) \frac{\partial C_{i-10}(u, \nu, w, \tau)}{\partial \nu} d w d \nu d u d \tau
\end{aligned}
$$




$$
\begin{aligned}
& -\frac{2 \pi}{L_{x} L_{y} L_{z}^{2}} \sum_{n=1}^{\infty} n F_{n C} e_{n C}(t) c_{n}(x) c_{n}(y) c_{n}(z) \int_{0}^{t} e_{n C}(-\tau) \int_{0}^{L_{x}} c_{n}(u) \\
& \times \int_{0}^{L_{y}} c_{n}(\nu) \int_{0}^{L_{z}} s_{n}(\nu) g_{L}(u, \nu, w, T) \\
& \times \frac{\partial C_{i-10}(u, \nu, w, \tau)}{\partial w} d w d \nu d u d \tau, i \geq 1, \\
& C_{01}(x, y, z, t)=-\frac{2 \pi}{L_{x}^{2} L_{y} L_{z}} \sum_{n=1}^{\infty} n F_{n C} c_{n}(x) c_{n}(y) c_{n}(z) e_{n C}(t) \int_{0}^{t} e_{n C}(-\tau) \int_{0}^{L_{x}} s_{n}(u) \\
& \times \int_{0}^{L_{y}} c_{n}(\nu) \int_{0}^{L_{z}} c_{n}(w) \frac{C_{00}^{\gamma}(u, \nu, w, \tau)}{P^{\gamma}(u, \nu, w, T)} \frac{\partial C_{00}(u, \nu, w, \tau)}{\partial u} d w d \nu d u d \tau \\
& -\frac{2 \pi}{L_{x} L_{y}^{2} L_{z}} \sum_{n=1}^{\infty} n F_{n C} c_{n}(x) c_{n}(y) c_{n}(z) e_{n C}(t) \int_{0}^{t} e_{n C}(-\tau) \int_{0}^{L_{x}} c_{n}(u) \\
& \times \int_{0}^{L_{y}} s_{n}(\nu) \int_{0}^{L_{z}} c_{n}(w) \frac{C_{00}^{\gamma}(u, \nu, w, \tau)}{P^{\gamma}(u, \nu, w, T)} \frac{\partial C_{00}(u, \nu, w, \tau)}{\partial \nu} d w d \nu d u d \tau \\
& -\frac{2 \pi}{L_{x} L_{y} L_{z}^{2}} \sum_{n=1}^{\infty} n e_{n C}(t) F_{n C} c_{n}(x) c_{n}(y) c_{n}(z) \int_{0}^{t} e_{n C}(-\tau) \int_{0}^{L_{x}} c_{n}(u) \\
& \times \int_{0}^{L_{y}} c_{n}(\nu) \int_{0}^{L_{z}} s_{n}(w) \frac{C_{00}^{\gamma}(u, \nu, w, \tau)}{P^{\gamma}(u, \nu, w, T)} \frac{\partial C_{00}(u, \nu, w, \tau)}{\partial w} d w d \nu d u d \tau
\end{aligned}
$$




$$
\begin{aligned}
& C_{02}(x, y, z, t)=-\frac{2 \pi}{L_{x}^{2} L_{y} L_{z}} \sum_{n=1}^{\infty} n F_{n C} c_{n}(x) c_{n}(y) c_{n}(z) e_{n C}(t) \int_{0}^{t} e_{n C}(-\tau) \int_{0}^{L_{x}} s_{n}(u) \\
& \times \int_{0}^{L_{y}} c_{n}(\nu) \int_{0}^{L_{z}} c_{n}(w) C_{01}(u, v, w, \tau) \frac{C_{00}^{\gamma-1}(u, \nu, w, \tau)}{P^{\gamma}(u, \nu, w, T)} \\
& \times \frac{\partial C_{00}(u, \nu, w, \tau)}{\partial u} d w d \nu d u d \tau \\
& -\frac{2 \pi}{L_{x} L_{y}^{2} L_{z}} \sum_{n=1}^{\infty} F_{n C} c_{n}(x) c_{n}(y) n c_{n}(z) e_{n C}(t) \int_{0}^{t} e_{n C}(-\tau) \int_{0}^{L_{x}} c_{n}(u) \\
& \times \int_{0}^{L_{y}} s_{n}(\nu) \int_{0}^{L_{z}} C_{01}(u, \nu, w, \tau) \frac{C_{00}^{\gamma-1}(u, \nu, w, \tau)}{P^{\gamma}(u, \nu, w, T)} \\
& \times \frac{\partial C_{00}(u, \nu, w, \tau)}{\partial \nu} c_{n}(w) d w d \nu d u d \tau \\
& -\frac{2 \pi}{L_{x} L_{y} L_{z}^{2}} \sum_{n=1}^{\infty} n F_{n C} c_{n}(x) c_{n}(y) c_{n}(z) e_{n C}(t) \int_{0}^{t} e_{n C}(-\tau) \int_{0}^{L_{x}} c_{n}(u) \\
& \times \int_{0}^{L_{y}} c_{n}(\nu) \int_{0}^{L_{z}} s_{n}(w) C_{01}(u, v, w, \tau) \frac{C_{00}^{\gamma-1}(u, \nu, w, \tau)}{P^{\gamma}(u, \nu, w, T)} \\
& \times \frac{\partial C_{00}(u, \nu, w, \tau)}{\partial w} d w d \nu d u d \tau \\
& -\frac{2 \pi}{L_{x}^{2} L_{y} L_{z}} \sum_{n=1}^{\infty} n c_{n}(x) F_{n C} c_{n}(y) c_{n}(z) e_{n C}(t) \int_{0}^{t} e_{n C}(-\tau) \int_{0}^{L_{x}} s_{n}(u) \\
& \times \int_{0}^{L_{y}} c_{n}(\nu) \int_{0}^{L_{z}} c_{n}(w) C_{01}(u, \nu, w, \tau) \frac{\partial C_{00}(u, \nu, w, \tau)}{\partial u} \\
& \times \frac{C_{00}^{\gamma-1}(u, \nu, w, \tau)}{P^{\gamma}(u, \nu, w, T)} d w d \nu d u d \tau
\end{aligned}
$$




$$
\begin{aligned}
& -\frac{2 \pi}{L_{x} L_{y}^{2} L_{z}} \sum_{n=1}^{\infty} n F_{n C} c_{n}(x) c_{n}(y) c_{n}(z) e_{n C}(t) \int_{0}^{t} e_{n C}(-\tau) \int_{0}^{L_{x}} c_{n}(u) \\
& \times \int_{0}^{L_{y}} s_{n}(\nu) \int_{0}^{L_{z}} c_{n}(w) C_{01}(u, \nu, w, \tau) \frac{C_{00}^{\gamma-1}(u, \nu, w, \tau)}{P^{\gamma}(u, \nu, w, T)} \\
& \times \frac{\partial C_{00}(u, \nu, w, \tau)}{\partial \nu} d w d \nu d u d \tau \\
& -\frac{2 \pi}{L_{x} L_{y} L_{z}^{2}} \sum_{n=1}^{\infty} n F_{n C} c_{n}(x) c_{n}(y) c_{n}(z) e_{n C}(t) \int_{0}^{t} e_{n C}(-\tau) \int_{0}^{L_{x}} c_{n}(u) \\
& \times \int_{0}^{L_{y}} c_{n}(\nu) \int_{0}^{L_{z}} s_{n}(w) C_{01}(u, \nu, w, \tau) \frac{C_{00}^{\gamma-1}(u, \nu, w, \tau)}{P^{\gamma}(u, \nu, w, T)} \\
& \times \frac{\partial C_{00}(u, \nu, w, \tau)}{\partial w} d w d \nu d u d \tau \\
& -\frac{2 \pi}{L_{x}^{2} L_{y} L_{z}} \sum_{n=1}^{\infty} F_{n C} c_{n}(x) c_{n}(y) c_{n}(z) e_{n C}(t) \int_{0}^{t} e_{n C}(-\tau) \int_{0}^{L_{x}} s_{n}(u) n \\
& \times \int_{0}^{L_{y}} c_{n}(\nu) \int_{0}^{L_{z}} c_{n}(w) \frac{C_{00}^{\gamma}(u, \nu, w, \tau)}{P^{\gamma}(u, \nu, w, T)} \frac{\partial C_{01}(u, \nu, w, \tau)}{\partial u} d w d \nu d u d \tau \\
& -\frac{2 \pi}{L_{x} L_{y}^{2} L_{z}} \sum_{n=1}^{\infty} c_{n}(x) e_{n C}(t) F_{n C} c_{n}(y) \int_{0}^{t} e_{n C}(-\tau) \int_{0}^{L_{x}} c_{n}(u) \\
& \times \int_{0}^{L_{y}} s_{n}(\nu) \int_{0}^{L_{z}} c_{n}(w) \frac{C_{00}^{\gamma}(u, \nu, w, \tau)}{P^{\gamma}(u, \nu, w, T)} \frac{\partial C_{01}(u, \nu, w, \tau)}{\partial \nu} d w d \nu d u d \tau \\
& \times n c_{n}(z)-\frac{2 \pi}{L_{x} L_{y} L_{z}^{2}} \sum_{n=1}^{\infty} n F_{n C} c_{n}(x) c_{n}(y) c_{n}(z) e_{n C}(t) \int_{0}^{t} e_{n C}(-\tau)
\end{aligned}
$$




$$
\begin{aligned}
& \times \int_{0}^{L_{x}} c_{n}(u) \int_{0}^{L_{y}} c_{n}(\nu) \int_{0}^{L_{z}} s_{n}(w) \frac{C_{00}^{\gamma}(u, \nu, w, \tau)}{P^{\gamma}(u, \nu, w, T)} \\
& \times \frac{\partial C_{01}(u, \nu, w, \tau)}{\partial w} d w d \nu d u d \tau, \\
& C_{11}(x, y, z, t)=-\frac{2 \pi}{L_{x}^{2} L_{y} L_{z}} \sum_{n=1}^{\infty} n F_{n C} c_{n}(x) c_{n}(y) c_{n}(z) e_{n C}(t) \int_{0}^{t} e_{n C}(-\tau) \int_{0}^{L_{x}} s_{n}(u) \\
& \times \int_{0}^{L_{y}} c_{n}(\nu) \int_{0}^{L_{z}} c_{n}(w) g_{L}(u, \nu, w, T) \frac{\partial C_{01}(u, \nu, w, \tau)}{\partial u} d w d \nu d u d \tau \\
& -\frac{2 \pi}{L_{x} L_{y}^{2} L_{z}} \sum_{n=1}^{\infty} n F_{n C} c_{n}(x) c_{n}(y) c_{n}(z) e_{n C}(t) \int_{0}^{t} e_{n C}(-\tau) \int_{0}^{L_{x}} c_{n}(u) \\
& \times \int_{0}^{L_{y}} s_{n}(\nu) \int_{0}^{L_{z}} c_{n}(w) g_{L}(u, \nu, w, T) \frac{\partial C_{01}(u, \nu, w, \tau)}{\partial \nu} d w d \nu d u d \tau \\
& -\frac{2 \pi}{L_{x} L_{y} L_{z}^{2}} \sum_{n=1}^{\infty} n e_{n C}(t) \int_{0}^{t} e_{n C}(-\tau) \int_{0}^{L_{x}} c_{n}(u) \int_{0}^{L_{y}} c_{n}(\nu) \int_{0}^{L_{z}} s_{n}(w) \\
& \times g_{L}(u, \nu, w, T) \frac{\partial C_{01}(u, \nu, w, \tau)}{\partial w} d w d \nu d u d \tau \\
& \times F_{n C} c_{n}(x) c_{n}(y) c_{n}(z)-\frac{2 \pi}{L_{x}^{2} L_{y} L_{z}} \sum_{n=1}^{\infty} F_{n C} c_{n}(x) c_{n}(y) c_{n}(z) e_{n C}(t) \\
& \times \int_{0}^{t} e_{n C}(-\tau) \int_{0}^{L_{x}} s_{n}(u) \int_{0}^{L_{y}} c_{n}(\nu) n \int_{0}^{L_{z}} c_{n}(w) \frac{C_{00}^{\gamma}(u, \nu, w, \tau)}{P^{\gamma}(u, \nu, w, T)} \\
& \times \frac{\partial C_{10}(u, \nu, w, \tau)}{\partial u} d w d \nu d u d \tau \\
& -\frac{2 \pi}{L_{x} L_{y}^{2} L_{z}} \sum_{n=1}^{\infty} n F_{n C} c_{n}(x) c_{n}(y) c_{n}(z) e_{n C}(t) \int_{0}^{t} e_{n C}(-\tau) \int_{0}^{L_{x}} c_{n}(u)
\end{aligned}
$$




$$
\begin{aligned}
& \times \int_{0}^{L_{y}} s_{n}(\nu) \int_{0}^{L_{z}} c_{n}(w) \frac{C_{00}^{\gamma}(u, \nu, w, \tau)}{P^{\gamma}(u, \nu, w, T)} \frac{\partial C_{10}(u, \nu, w, \tau)}{\partial \nu} d w d \nu d u d \tau \\
& -\frac{2 \pi}{L_{x} L_{y} L_{z}^{2}} \sum_{n=1}^{\infty} n F_{n C} c_{n}(x) c_{n}(y) c_{n}(z) e_{n C}(t) \int_{0}^{t} e_{n C}(-\tau) \int_{0}^{L_{x}} c_{n}(u) \\
& \times \int_{0}^{L_{y}} c_{n}(\nu) \int_{0}^{L_{z}} s_{n}(w) \frac{C_{00}^{\gamma}(u, \nu, w, \tau)}{P^{\gamma}(u, \nu, w, T)} \frac{\partial C_{10}(u, \nu, w, \tau)}{\partial w} d w d \nu d u d \tau \\
& -\frac{2 \pi}{L_{x}^{2} L_{y} L_{z}} \sum_{n=1}^{\infty} n F_{n C} c_{n}(x) c_{n}(y) c_{n}(z) e_{n C}(t) \int_{0}^{t} e_{n C}(-\tau) \int_{0}^{L_{x}} s_{n}(u) \\
& \times \int_{0}^{L_{y}} c_{n}(\nu) \int_{0}^{L_{z}} c_{n}(w) C_{10}(u, \nu, w, \tau) \frac{C_{00}^{\gamma-1}(u, \nu, w, \tau)}{P^{\gamma}(u, \nu, w, T)} \\
& \times \frac{\partial C_{00}(u, \nu, w, \tau)}{\partial u} d w d \nu d u d \tau \\
& -\frac{2 \pi}{L_{x} L_{y}^{2} L_{z}} \sum_{n=1}^{\infty} n F_{n C} c_{n}(x) c_{n}(y) c_{n}(z) e_{n C}(t) \int_{0}^{t} e_{n C}(-\tau) \int_{0}^{L_{x}} c_{n}(u) \\
& \times \int_{0}^{L_{y}} S_{n}(\nu) \int_{0}^{L_{z}} c_{n}(w) \frac{C_{00}^{\gamma-1}(u, \nu, w, \tau)}{P^{\gamma}(u, \nu, w, T)} \\
& \times \frac{\partial C_{00}(u, \nu, w, \tau)}{\partial \nu} C_{10}(u, \nu, w, \tau) d w d \nu d u d \tau \\
& -\frac{2 \pi}{L_{x} L_{y} L_{z}^{2}} \sum_{n=1}^{\infty} n F_{n C} c_{n}(x) c_{n}(y) c_{n}(z) e_{n C}(t) \int_{0}^{t} e_{n C}(-\tau) \int_{0}^{L_{x}} c_{n}(u) \\
& \times \int_{0}^{L_{y}} c_{n}(\nu) \int_{0}^{L_{z}} s_{n}(w) C_{10}(u, \nu, w, \tau) \frac{C_{00}^{\gamma-1}(u, \nu, w, \tau)}{P^{\gamma}(u, \nu, w, T)} \\
& \times \frac{\partial C_{00}(u, \nu, w, \tau)}{\partial w} d w d \nu d u d \tau .
\end{aligned}
$$

